\title{
Quantum Gravity Phenomenology Induced in the Propagation of UHECR, a Kinematical Solution in Finsler and Generalized Finsler Spacetime
}

\author{
Marco Danilo Claudio Torri 1,2 (D) \\ 1 Dipartimento di Fisica, Università degli Studi di Napoli, Complesso Univ. Monte S. Angelo, \\ 80126 Napoli, Italy; marco.torri@mi.infn.it or marco.torri@unimi.it \\ 2 Dipartimento di Fisica, Università degli Studi di Milano and INFN Sezione di Milano, Via Celoria 16, \\ 20133 Milano, Italy
}

check for updates

Citation: Torri, M.D.C. Quantum Gravity Phenomenology Induced in the Propagation of UHECR, a Kinematical Solution in Finsler and Generalized Finsler Spacetime. Galaxies 2021, 9, 103. https://doi.org/ 10.3390 /galaxies 9040103

Academic Editor: Marco Schreck

Received: 30 September 2021

Accepted: 10 November 2021

Published: 14 November 2021

Publisher's Note: MDPI stays neutral with regard to jurisdictional claims in published maps and institutional affiliations.

Copyright: (C) 2021 by the authors. Licensee MDPI, Basel, Switzerland. This article is an open access article distributed under the terms and conditions of the Creative Commons Attribution (CC BY) license (https:// creativecommons.org/licenses/by/ $4.0 /)$.
Abstract: It is well-known that the universe is opaque to the propagation of Ultra-High-Energy Cosmic Rays (UHECRs) since these particles dissipate energy during their propagation interacting with the background fields present in the universe, mainly with the Cosmic Microwave Background $(\mathrm{CMB})$ in the so-called GZK cut-off phenomenon. Some experimental evidence seems to hint at the possibility of a dilation of the GZK predicted opacity sphere. It is well-known that kinematical perturbations caused by supposed quantum gravity (QG) effects can modify the foreseen GZK opacity horizon. The introduction of Lorentz Invariance Violation can indeed reduce, and in some cases making negligible, the CMB-UHECRs interaction probability. In this work, we explore the effects induced by modified kinematics in the UHECR lightest component phenomenology from the QG perspective. We explore the possibility of a geometrical description of the massive fermions interaction with the supposed quantum structure of spacetime in order to introduce a Lorentz covariance modification. The kinematics are amended, modifying the dispersion relations of free particles in the context of a covariance-preserving framework. This spacetime description requires a more general geometry than the usual Riemannian one, indicating, for instance, the Finsler construction and the related generalized Finsler spacetime as ideal candidates. Finally we investigate the correlation between the magnitude of Lorentz covariance modification and the attenuation length of the photopion production process related to the GZK cut-off, demonstrating that the predicted opacity horizon can be dilated even in the context of a theory that does not require any privileged reference frame.

Keywords: quantum gravity; lorentz invariance violation; doubly special relativity; finsler geometry; astroparticle physics; cosmic rays

\section{Introduction}

The universe is opaque to the propagation of radiation in particular of its most energetic component, that is cosmic rays (CRs). This part of the extraterrestrial radiation is composed of charged particles, protons and heavier (bare) nuclei, most likely of extragalactic origin. Since the universe opacity is more pronounced at the highest energies, in this work we deal with Ultra High Energy Cosmic Rays (UHECRs) that is the most energetic fraction of CR with energies larger than $\sim 10^{18} \mathrm{eV}$. CRs during their propagation may interact with background radiation fields in particular with the Extragalactic Background Light (EBL) and at sufficiently high energies with the Cosmic Microwave Background (CMB) being attenuated in a way that depends on their energy and nature. The UHECR's lightest component, protons with energies above the photopion threshold $\sim 5 \times 10^{19} \mathrm{eV}$, dissipate energy mainly via pair production or photopion creation, instead the heavier component composed of bare nuclei can also undergo photodissociation processes. A $\mathrm{CR}$ can be generated with a limited energy proportional to the product of the magnetic field and the dimension of any candidate source: $E \sim B \times d$, following the so-called Hillas 
criterion [1]. Since UHECRs' free propagation path is finite and their energy is limited, they can be detected on Earth only under a determined energy threshold if they propagate for long enough distances. This phenomenon poses an upper limit on the detectable UHECRs energy and in the case of the lightest component is named GZK cut-off from the name of the physicists Greisen, Zatsepin and Kuzmin [2,3].

The physics correlated with the UHECR propagation can be exploited to investigate new phenomena, such as the supposed quantum structure of the spacetime [4-9]. Astroparticle physics can be a useful framework to conduct searches for possible departures from the Lorentz covariance since in some theoretical models the quantum gravity (QG) effects are supposed to be more visible in the high-energy limit as Lorentz Invariance Violation (LIV) effects. UHECRs propagate for cosmic distances and reach extremely high energies, therefore they can open a window on the Planck scale physics.

Currently, there is no definitive theory that unifies quantum physics with gravity. The greatest challenge in formulating such a unified theory is the actual impossibility of obtaining the energies needed to probe the Planck scale realm. It is commonly believed that the Planck energy $E_{P}=\sqrt{\hbar c^{5} G^{-1}} \simeq 1.22 \times 10^{19} \mathrm{GeV}$ represents the threshold that separates the classical formulation of physics, which is General Relativity (GR), from the quantum realm, represented by Quantum Field Theory (QFT). Nevertheless some QG signatures may become manifest in a low-energy regime as residual effects and these perturbations of standard physics may give access to the possible phenomenology induced by QG.

In this work, we investigate the possibility to detect QG signatures as departures from the Lorentz covariance in the UHECR sector. Indeed, the introduction of QG motivated modifications could modify the threshold of physical processes in UHECR physics, influencing the generation of hadronic showers in the atmosphere at the detection. Moreover, QG can perturb at sufficiently high energies the free particle kinematics reducing the interaction with the background fields, mainly the $\mathrm{CMB}$, and the related energy dissipation caused by the GZK effect and photodissociation process, resulting in an enlargement of the volume of the universe accessible at UHECRs. A natural way of looking for QG signals involves the determination of UHECR propagation length. This result can be obtained by finding out the source of these particles, for instance, detecting anisotropies in their flux. Indeed, anisotropy searches will require a better knowledge of the supposed UHECR sources distribution.

The comprehension of UHECR physics must be improved in order to conduct the anisotropy analysis that underlies all the potential QG studies in the CR propagation sector. Indeed, this analysis can be spoiled by the presence of Galactic (GMF) and Extragalactic Magnetic Fields (EGMF). Since CR are charged particles, their trajectories are affected by magnetic fields and the measure of the deflection can be obtained evaluating the particle rigidity, that is the ratio of $C R$ energy and charge: $R=E / Z$, for higher rigidity values the resulting deflections are smaller: $\langle\theta\rangle \sim 1 / R$, where $\langle\theta\rangle$ is the average deflection angle. The theoretical and experimental uncertainties about EBL, GMF [10,11] and particularly EGMF can spoil this analysis. Posing constraints on the magnitude of EGMF, for instance, is extremely complicated since the universe is mostly composed of cosmic void, therefore the EGMF magnitude evaluation ranges from $\sim \times 10^{-19} \mathrm{G}$ [12] to $\sim \times 10^{-19} \mathrm{G}$ [13]. Most of the studies about EGMF effects on UHECR propagation are conducted by simulating the cosmological structure formation, but with conflicting and inconclusive results $[14,15]$. A last remark about the necessity of better understanding the nature of the primary $\mathrm{CR}$ in order to discriminate the lighter UHECR component from the heavier one on a particle-by-particle basis. Recent studies of the Pierre Auger collaboration indicate that above the energy threshold of $\sim \times 10^{18} \mathrm{eV}$ the $\mathrm{CR}$ flux is dominated by the light primaries, nevertheless as the energy increases the lightest fraction (protons) seems to be partially replaced by an intermediate mass component (He-N) with a Fe contribution above $\sim \times 10^{19.4} \mathrm{eV}[16,17]$.

The phenomenological analysis conducted to detect the presumed QG effects in the present study is limited to the lightest component of the UHECRs, that is protons. This 
component is interesting since it is the least deflected one by magnetic fields during propagation. In fact, UHE protons can reach rigidities beyond $\sim 20 \times 10^{18} \mathrm{eV}$ presenting ballistic trajectories, which can allow for small-scale anisotropies searches.

For a more exhaustive and complete introduction on the CR physics and the related issues, we suggest the lecture of the review [18].

There are many theoretical models that investigate the possibility of modifications as QG-induced perturbations [4,19-25], but we conduct our investigation in the context of Homogeneously Modified Special Relativity (HMSR) [26]. In this theoretical framework the covariance is modified and not broken as in Doubly Special Relativity (DSR) [22-25]. Hence, the kinematical symmetry is again valid in an amended formulation and the introduction of a privileged reference frame is not required. In this context a minimal extension of the Standard Model (SM) of particle physics can be formulated preserving the modified covariance. The QG perturbations are introduced in the free particle kinematics modifying the dispersion relations (DRs). This idea is motivated by the hypothesis that the effects induced by the quantum structure of the background can be geometrized requiring a more general structure to set the theory than the usual Riemann geometry, that is the Finsler one. The introduction of QG kinematical perturbations can modify the allowed phase space for the interaction with the $\mathrm{CMB}$ and the pion creation process. The phenomenological effects are evaluated via numerical simulations conducted using an ad hoc version of the software SimProp [27], modified in order to include the QG kinematical perturbations [8]. The introduction of Lorentz covariance modifications induce an opacity horizon enlargement $[7,8]$, as foreseen even in the case of pure LIV [4-6].

This work is structured as follows: first we introduce the theoretical framework motivated by the QG perspective, geometrizing the interaction of a free particle with the background. Then, we introduce a minimal extension of the Standard Model of particle physics in an amended covariant formulation. In the following we introduce the physics related with the UHECRs propagation explaining where the QG effects can manifest themselves. Afterwards, we present the numerical results obtained via the modified SimProp software. Finally, we analyze the results with particular emphasis on the possibility of obtaining detectable phenomenological effects in this sector in the context of a covariant framework, that retains a modified version of the kinematical symmetry group (the Lorentz/Poincaré one).

\section{Kinematical Modifications in an Isotropy Preserving Scenario}

In this work, we explore the possibility of investigating the presumed QG effects perturbing the free particle kinematics and we obtain a minimal extension of the particle $\mathrm{SM}$ in a framework that preserves an amended covariance formulation [26].

\section{Modified Dispersion Relations}

The QG-caused perturbations are introduced only in the free particle kinematics in order to prevent the introduction of any exotic particle or reaction. As a reasonable physical hypothesis only the Dispersion Relations (DRs) of massive fermions are modified, because of the gravitational nature of these supposed effects, as done in the great part of theories $[4,19,21-25,28,29]$ that tackle the Special Relativity (SR) modifications from a QG perspective. In this work, every particle species is supposed to have its personal Modified Dispersion Relation (MDR):

$$
F^{2}(p)=E^{2}\left(1+h\left(\frac{|\vec{p}|}{E}\right)\right)-|\vec{p}|^{2}\left(1+k\left(\frac{|\vec{p}|}{E}\right)\right)=m^{2}
$$

where besides the particle energy $E$, momentum $\vec{p}$ and mass $m$ the QG perturbations are encoded in the peculiar functions $h$ and $k$ depending on the particle species. These functions have to satisfy the perturbation condition:

$$
|h| \ll 1 \quad|k| \ll 1 \quad h, k \simeq O(1),
$$


with at least first and second order derivatives limited with the same perturbative magnitude. One may consider, for instance, the linear function $\alpha(|\vec{p}| / E)$ or the translated exponential $\exp (\alpha|\vec{p}| / E)-1$ with $\alpha$ a strongly limited coefficient that encodes the perturbation character of the selected function. In the following, all the terms with comparable magnitude will be considered first-order perturbations (See Appendix A) $\simeq O(1)$.

The perturbation functions $h$ and $k$ are 0-degree homogeneous and depend on the 3momentum magnitude $|\vec{p}|$ in order to be rotationally invariant, as conjectured in [26]. This perturbation choice can guarantee the MDR geometrical origin, in this way the function $F^{2}(p)$ is 2-homogeneous and can be a candidate Finsler pseudo-norm. It is important to emphasize that we are dealing with a pseudo-Finsler geometry since the metric signature is not positive definite, in the sense that the underlying structure is the Minkowski geometry. As it will be clear in the following, if the perturbation functions are chosen as $h=k$ one can obtain a conformally flat momentum space and if $h>k$ the MDR Equation (1) can be approximated with the MDR defined in [26]:

$$
F^{2}(p)=E^{2}-|\vec{p}|^{2}\left(1-f\left(\frac{|\vec{p}|}{E}\right)\right)=m^{2},
$$

with $f \simeq(h-k)$. Another important feature emerges thanks to the dependence of the perturbations on the ratio $|\vec{p}| / E$, indeed with the latter MDR choice:

$$
\begin{aligned}
& \frac{|\vec{p}|}{E} \rightarrow(1+\delta) \text { for } E \rightarrow \infty \text { with } 0<\delta \ll 1 \\
& f\left(\frac{|\vec{p}|}{E}\right) \rightarrow \epsilon \ll 1
\end{aligned}
$$

and from this the possibility of obtaining in the high-energy limit the first formulation of Very Special Relativity (VSR) follows, and if $f=h-k \neq 0$ Equation (1). Every particle admits, therefore, its personal Maximum Attainable Velocity (MAV) as supposed by Coleman and Glashow [4]:

$$
c^{\prime}=\left(1-f\left(\frac{|\vec{p}|}{E}\right)\right) \rightarrow(1-\epsilon) .
$$

This feature is present in VSR and HMSR and as a direct consequence a rich phenomenology can be obtained in different physical sectors, for instance, in neutrino oscillation physics [30,31].

\section{Finsler Geometry}

We give now a brief introduction to Finsler geometry that will be useful in the following. A Finsler geometric structure can be defined as a manifold $M$ where in every tangent space a norm function $F$ is defined not necessarily starting from an inner product. $F$ is a positively homogeneous norm only if the Hessian of $F^{2}$ is positive definite. The norm must be a real and positive definite function of the section of the tangent space $T_{x} M$, which depends on the point $x$ and on a vector $v \in T_{x} M$. The norm must be 1-degree homogeneous with respect to the vectors satisfying the relations:

- $F:\left(M, T_{x} M\right) \rightarrow \mathbb{R}^{+}$

- $F(x, v)>0 \quad \forall v \in T_{x} M, v \neq 0, \forall x \in M$

- $F(x, \lambda v)=|\lambda| F(x, v)$.

In Finsler geometry the norm can define a local metric via the equation:

$$
g_{\mu v}=\frac{1}{2} \frac{\partial^{2} F(x, v)^{2}}{\partial v^{\mu} \partial v^{v}},
$$


which requires that $\operatorname{det} g_{\mu \nu} \neq 0$ and $g_{\mu \nu} \in \mathbb{R} \forall \mu, v$. In this way, it is possible to reobtain a vector norm:

$$
F(x, v)=\sqrt{g(x, v) \mu v v^{\mu} v^{v}} .
$$

As in Riemann geometry it is possible to define a duality relation between vectors and dual forms defining the Legendre transform. Using the metric the resulting bijection is given by:

$$
\begin{aligned}
& l: T_{x} M \rightarrow T_{x}^{*} M \\
& l(v)_{\mu}=\omega_{\mu}=g(x, v)_{\mu v} v^{v} .
\end{aligned}
$$

The previous definition is valid for a Finsler structure with a definite signature of the metric, but it must be generalized in order to deal with the physical spacetime, which exhibits a Minkowski-type underlying metric of indefinite-signature. A systematical introduction to this issue and the so-called pseudo-Finsler geometry can be found in [32-37]. Here, we follow the definition given in $[33,35]$ and pose a pseudo-Finsler geometric structure modifying the previous definition and requiring that:

- $\quad$ there exists a connected component $T$ of the pre-image of $F^{2} \subset T M$, such that on $T$ the metric defined by the Hessian exists, is smooth and has Lorentzian signature $(+,-,-,-)$.

A typical difference between Finsler and Riemann geometry consists in the necessity to consider the fiber bundle since in the first case objects exist on $T_{x} M$, whereas in the second, they do so on the manifold $M$. This fact implies that in Finsler geometry, vectors and covectors must be studied in $T(T M \backslash 0)$ and $T^{*}(T M \backslash 0)$, respectively. Moreover, it is necessary to introduce the horizontal-vertical decomposition and the related nonlinear connection for the $T(T M \backslash 0)$ and $T^{*}(T M \backslash 0)$ structures, as in [38].

Considering $T^{*}(T M \backslash 0)$, one can introduce the vertical distribution generated by the derivative $\partial / \partial v$ as:

$$
V: w \in T^{*} M \rightarrow V_{w} \subset T_{w}\left(T^{*} M\right)
$$

Given the vertical distribution, it is possible to identify a complementary structure, the horizontal distribution, named nonlinear connection, for which the Whitney decomposition is valid [39]:

$$
T_{w}\left(T^{*} M\right)=N_{w} \oplus V_{w} .
$$

The nonlinear connection is a collection of homogeneous functions of degree 1, locally defined on the manifold, such that:

$$
\frac{\delta}{\delta x^{\mu}}=\frac{\partial}{\partial x^{\mu}}+N_{\mu v}(x, p) \frac{\partial}{\partial p_{v}} .
$$

In this work, we follow [38] and as nonlinear connection we choose the usual General Relativity (GR) form:

$$
N_{\mu \nu}(x, p)=\Gamma_{\mu \nu}^{\alpha}(x) p_{\alpha},
$$

where $\Gamma_{\mu \nu}^{\alpha}$ represents the GR affine connection. The choice for a nonlinear connection is not uniquely defined, but this particular formulation is the most convenient one in this context.

Analogously, the cotangent space is spanned by the differential basis defined by:

$$
\delta p_{\mu}=\mathrm{d} p_{\mu}-N_{\mu \nu}(x, p) \mathrm{d} x^{v}
$$

In the context of Finsler geometry, it is possible to generalize the definition of the affine connection via the general Christoffel symbols:

$$
H_{\mu \nu}^{\alpha}(x, p)=\frac{1}{2} g^{\alpha \beta}(x, p)\left(\frac{\delta g_{\beta, \nu}(x, p)}{\delta x^{\mu}}+\frac{\delta g_{\mu, \beta}(x, p)}{\delta x^{v}}-\frac{\delta g_{\mu, v}(x, p)}{\delta x^{\beta}}\right)
$$


and the geodesic equations become:

$$
\frac{\mathrm{d}^{2} x^{\alpha}}{\mathrm{d} \tau^{2}}+H_{\mu \nu}^{\alpha} \frac{\mathrm{d} x^{\mu}}{\mathrm{d} \tau} \frac{\mathrm{d} x^{v}}{\mathrm{~d} \tau}=0 .
$$

The study conducted in this work can be generalized with these prescriptions in a curved spacetime. In the following, we will consider a geometry with no matter induced curvature for the investigation of the phenomenology introduced in the propagation of UHECRs. Hence, the nonlinear connection Equation (12) is zero and the derivative Equation (11) reduces to the usual partial derivative.

\section{Finsler Geometry and HMSR}

The MDR (1) can be interpreted as the Finsler pseudo-norm characterizing the spacetime (momentum space) structure. In HMSR, the standard physics is modified in a CPTeven scenario, the MDRs, indeed, do not exhibit any dependence on helicities, for instance, and are supposed to be equal for particles and the related antiparticles. A well-known result about fundamental physics symmetry states that in a CPT-odd scenario the Lorentz symmetry as usually formulated must be violated, whereas the opposite statement is not automatically true, and therefore it is possible to violate or modify covariance, while preserving the CPT symmetry [40,41]. A review on the implications of the CPT symmetry in particle and astroparticle physics and its relation to the Lorentz/Poincare symmetry is presented, for instance, in [42].

Following the idea presented in [26], the MDR Equation (1) are promoted to the role of a Finsler norm and the related momentum space metric is obtained as the Hessian of the squared norm:

$$
\widetilde{g}^{\mu \nu}(p)=\frac{1}{2} \frac{\partial}{\partial p_{\mu}} \frac{\partial}{\partial p_{v}} F^{2}(E, \vec{p}) .
$$

Since the function $F^{2}$ is 2-degree homogeneous, the computation produces a 0 homogeneous tensor that can be written as:

$$
\widetilde{g}^{\mu \nu}(p)=D_{4 \times 4}+A_{4 \times 4},
$$

with a diagonal matrix $D$ :

$$
D=\left(\begin{array}{cc}
(1+h(|\vec{p}| / E)) & \overrightarrow{0} \\
\overrightarrow{0}^{t} & -(1+k(|\vec{p}| / E)) \mathbb{I}_{3 \times 3}
\end{array}\right) .
$$

The matrix $D$ is the associated generalized Hamilton's space metric that effectively contributes to the computation of the MDR Equation (1). Indeed, by direct calculation it is straightforward to demonstrate the relation:

$$
p_{\mu} \widetilde{g}^{\mu v}(p) p_{v}=p_{\mu} D^{\mu v}(p) p_{v}=E^{2}\left(1+h\left(\frac{|\vec{p}|}{E}\right)\right)-|\vec{p}|^{2}\left(1+k\left(\frac{|\vec{p}|}{E}\right)\right) .
$$

The complete Finsler metric tensor is written including the matrix $A$ Equation (17) and satisfies the Finsler requirement of a totally symmetric associated Cartan's tensor:

$$
C_{\mu v \alpha}=\frac{1}{2} \frac{\partial^{3} F^{2}(p)}{\partial p_{\mu} \partial p_{\nu} \partial p_{\alpha}} .
$$

The matrix $A$ has both diagonal and nondiagonal entries and thanks to the explicit form acquired by the metric tensor $\widetilde{g}$ it is simple to find out that this matrix gives no contribution to the norm of a covector: $p_{\mu} A^{\mu v} p_{v}=0$ Equation (19). For the following computations, it is important to emphasize that the entries of this matrix are first-order perturbations under the assumption that the functions $h, k \simeq O(1)$ and their derivatives have a perturbative character See Appendix A, hence $A \simeq O(1)$. 
From the Finsler co-metric, one can derive the metric associated with coordinate space as the inverse one, via the defining equation:

$$
\widetilde{g}^{\mu \alpha} g_{\alpha v}=\delta_{v}^{\mu}
$$

obtaining the explicit form of the coordinate space metric:

$$
g_{\mu v}(p)=D^{*}-A_{4 \times 4}+O(2)
$$

and the diagonal matrix $D^{*}$ :

$$
D^{*}=\left(\begin{array}{cc}
(1-h(|\vec{p}| / E)) & \overrightarrow{0} \\
\overrightarrow{0}^{t} & -(1-k(|\vec{p}| / E)) \mathbb{I}_{3 \times 3}
\end{array}\right),
$$

where $O(2)$ indicates a second-order perturbation with respect to the scale fixed by the function $h$ and $k$ Equation (1).

\section{Lagrangian and Hamilton Finsler Geometry}

In this work, we present a precise insight of the existing correlation between coordinate and momentum space in the context of HMSR starting from the action:

$$
\begin{aligned}
S & =\int \mathcal{L} \mathrm{d} \tau=\int\left(\dot{x}^{\mu} p_{\mu}-\frac{\lambda}{2} F^{2}(p)\right) \mathrm{d} \tau \\
& =\int\left(\dot{x}^{\mu} p_{\mu}-\frac{\lambda}{2}\left(\widetilde{g}^{\alpha \beta}(p) p_{\alpha} p_{\beta}-m^{2}\right)\right) \mathrm{d} \tau,
\end{aligned}
$$

where the Lagrangian $\mathcal{L}$ is defined using a Lagrange multiplier $\lambda$ to pose the constraint that the MDR, $F^{2}(p)=m^{2}$, must be satisfied [43]. The action $S$ is obtained integrating the Lagrangian $\mathcal{L}$ with respect to the proper time $\tau$.

The equations of motion can be obtained varying the Lagrangian $\mathcal{L}$. Hence, one can obtain the partial derivatives with respect to the velocity $\dot{x}^{\mu}$ and the Lagrange multiplier $\lambda$ :

$$
\begin{aligned}
\frac{\partial \mathcal{L}}{\partial \dot{x}^{\mu}}=0 \Rightarrow \frac{\mathrm{d}}{\mathrm{d} \tau}\left(\frac{\partial \mathcal{L}}{\partial \dot{x}^{\mu}}\right)=0 \Rightarrow \frac{\mathrm{d} p_{\mu}}{\mathrm{d} \tau}=0 \\
\frac{\partial \mathcal{L}}{\partial \lambda}=0 \Rightarrow \widetilde{g}^{\mu \nu}(p) p_{\mu} p_{v}=m^{2} .
\end{aligned}
$$

The variation calculated with respect to the momentum $p_{\mu}$ gives:

$$
\frac{\partial \mathcal{L}}{\partial p_{\mu}}=0 \Rightarrow \dot{x}^{\mu}=\lambda \widetilde{g}^{\mu v}(p) p_{v}+\frac{\lambda}{2}\left(\frac{\partial \widetilde{g}^{\alpha \beta}(p)}{\partial p_{\mu}}\right) p_{\alpha} p_{\beta} .
$$

A simple application of the Euler's theorem on homogeneous functions implies that the second term on the right-hand side of the previous relation is zero thanks to the 0 homogeneity of the metric tensor. Therefore, the velocity can be written in the simple form:

$$
\dot{x}^{\mu}=\lambda \widetilde{g}^{\mu v}(p) p_{v}
$$

Inverting the previous relation, the momentum can be expressed as:

$$
p_{\mu}=\lambda^{-1} g_{\mu \nu} \dot{x}^{v} .
$$

The momentum can be expressed as a function of the velocity solving Equation (28), for instance, perturbatively. As a consequence, the coordinate space metric $\widetilde{g}_{\mu v}(p)$, defined as the inverse of the momentum space metric $\widetilde{g}^{\mu v}(p)$, can be written as a function of coordinate $x$ and velocity $\dot{x}$ :

$$
\tilde{g}_{\mu \nu}(p)=g_{\mu v}(\dot{x}) .
$$


The Lagrangian can be computed substituting Equation (26) in the action Equation (24):

$$
\mathcal{L}=\frac{1}{2 \lambda} g_{\mu \nu}(p) \dot{x}^{\mu} \dot{x}^{v}+\frac{\lambda}{2} m^{2}
$$

The variation of the previous relation with respect to the parameter $\lambda$ gives:

$$
\frac{\partial \mathcal{L}}{\partial \lambda}=0 \Rightarrow \frac{g_{\mu \nu}(\dot{x}) \dot{x}^{\mu} \dot{x}^{\nu}}{\lambda^{2}}=m^{2} \Rightarrow \lambda=\frac{\sqrt{g_{\mu \nu}(\dot{x}) \dot{x}^{\mu} \dot{x}^{v}}}{m}
$$

and then the Lagrangian can be written as:

$$
\mathcal{L}=m \sqrt{g_{\mu \nu}(\dot{x}) \dot{x}^{\mu} \dot{x}^{\nu}}
$$

From the Legendre transform of action Equation (24), it is possible to compute the energy $E$ solving the following equation for $p_{0}$ :

$$
\dot{\vec{x}} \cdot \dot{\vec{p}}-\mathcal{L}=0 \Rightarrow \widetilde{g}^{\mu v} p_{\mu} p_{v}-m^{2}=0
$$

It is important to underline that this equation is equivalent to the mass-shell constraint obtained from the MDR Equation (1). The energy $E$ can finally be considered as the Hamiltonian of the system:

$$
\mathcal{H}=E=p_{0}
$$

The relation between the Lagrangian and the Hamiltonian formulation has been established and it is possible to compute the norm for the velocity $\dot{x}$. This function is associated with the momentum space norm $F$ which is defined via the MDR Equation (1). The coordinate space norm can be determined starting from the metric Equation (22):

$$
\begin{aligned}
F(\dot{x})^{2} & =\dot{x}^{\mu} g_{\mu \nu}(\dot{x}) \dot{x}^{\nu}=\dot{x}^{\mu}\left(D_{\mu \nu}^{*}-A_{\mu \nu}+O(2)\right) \dot{x}^{\nu} \\
& =(1-h(|\vec{p}| / E)) \dot{x}_{0}^{2}-(1-k(|\vec{p}| / E)) \vec{x}^{2}+O(2) .
\end{aligned}
$$

As a final remark it is possible to state that the resulting structure is a Finsler geometry.

\section{HMSR Generalized Finsler Spacetime}

In the following we will consider a generalized Finsler geometry model to set the stage of the HMSR formulation. In this context, we will obtain an extension of the Standard Model (SM) of particle physics and will conduct the computations related with the GZK cutoff phenomenon.

The generalized Finsler geometry is a less restrictive structure which does not require a totally symmetric Cartan's tensor associated to the metric Equation (20), therefore, the construction of the geometry is easier. In this context the spacetime and the momentum metrics Equations (17) and (22) can be simplified taking into account only the diagonal parts Equations (18) and (23):

$$
\begin{aligned}
& g_{\mu \nu}(p)=D=\left(\begin{array}{cc}
(1+h(|\vec{p}| / E)) & \overrightarrow{0} \\
\overrightarrow{0}^{t} & -(1+k(|\vec{p}| / E)) \mathbb{I}_{3 \times 3}
\end{array}\right) \\
& g^{\mu \nu}(p)=D^{*}=\left(\begin{array}{cc}
(1-h(|\vec{p}| / E)) & \overrightarrow{0} \\
\overrightarrow{0}^{t} & -(1-k(|\vec{p}| / E)) \mathbb{I}_{3 \times 3}
\end{array}\right) .
\end{aligned}
$$

In this way, the coordinate and momentum metrics are reduced to the parts that really contribute in evaluating the squared norm of a vector or a covector, respectively.

Starting from the defining equations: 


$$
\begin{aligned}
& g_{\mu v}(p)=e_{\mu}^{a}(p) \eta_{a b} e_{v}^{b}(p) \\
& \widetilde{g}^{\mu v}(p)=e_{a}^{\mu}(p) \eta^{a b} e_{b}^{v}(p) .
\end{aligned}
$$

it is possible to compute the generalized associated vierbein, which can be written as:

$$
\begin{aligned}
& e_{\mu}^{a}(p)=\left(\begin{array}{cc}
\sqrt{1+h((|\vec{p}| / E))} & \overrightarrow{0} \\
\overrightarrow{0}^{t} & \sqrt{1+k((|\vec{p}| / E))} \mathbb{I}_{3 \times 3}
\end{array}\right) \\
& e_{a}^{\mu}(p)=\left(\begin{array}{cc}
\sqrt{1-h((|\vec{p}| / E))} & \overrightarrow{0} \\
\overrightarrow{0}^{t} & \sqrt{1-k((|\vec{p}| / E))} \mathbb{I}_{3 \times 3}
\end{array}\right) .
\end{aligned}
$$

It is simple to determine the perturbative order $O(1)$ of part of these matrix entries using the approximation $\sqrt{1 \pm \epsilon} \simeq 1 \pm \frac{1}{2} \epsilon$, valid for $\epsilon \ll 1$.

The form of the vierbein Equation (39) will be used in all the following computations, for instance, when evaluating the kinematical invariants (the Mandelstam $s$ variable of the reactions involved in the GZK cut-off phenomenon).

\subsection{Generalized Covariance}

In the HMSR model [26] every particle has its personal modified spacetime, which is parameterized by the particle momentum. This means that every physical quantity related with a given particle is generalized and lives in a spacetime that acquires an explicit dependence on the particle energy. It is therefore necessary to introduce an original formalism to correlate different local spaces, using the generalized vierbein elements as projectors from every spacetime to a common flat Minkowski support space. Here, we report a scheme of how the correlation between different local spaces is established:

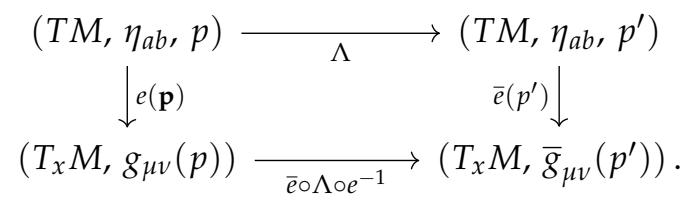

The Greek indices refer to the local curved geometric structures, whereas the Latin ones refer to the common Minkowski support space. Referring to the previous scheme, using the vierbein elements as projectors, the generalized Lorentz transformations can be obtained:

$$
\Lambda_{\mu}^{v}(p)=e_{\mu}^{a}(\Lambda p) \Lambda_{a}^{b} e_{v}^{b}(p),
$$

where the elements $\Lambda_{a}^{b}$ belong to the Lorentz group and are defined in the flat Minkowski spacetime and the used vierbein is defined in Equation (39) and includes all terms up to the perturbative order under consideration. The Lorentz covariance is promoted to a diffeomorphism invariance and the introduced class of Modified Lorentz Transformations (MLT) represent the isometries of the MDR of Equation (1). We point out that the previous construction about the generalization of covariance is valid if the spacetime is metrizable and admits a vierbein, hence the result is valid even in different contexts. Therefore, the present prescription can be used to pose analogous definitions and set the stage of the model in the context of the more restrictive Finsler geometry.

\subsection{Affine and Spinorial Connections}

The geometrical structure is characterized by the affine and the spinorial connections. In Finsler geometry, the affine connection, that is the Christoffel symbol, can be defined using Equation (14). In the case of zero spacetime curvature, the nonlinear connection 
Equation (12) is $N_{\mu v}=0$ and therefore the derivative Equation (14) reduces to the ordinary one:

$$
\frac{\delta}{\delta x^{\mu}}=\frac{\partial}{\partial x^{\mu}} .
$$

Since in the case of absence of curvature, the metric depends only on the momentum (or equivalently on the velocity) and not on the coordinates, the Christoffel symbol Equation (14) becomes:

$$
H_{\mu \nu}^{\alpha}(x, p)=\frac{1}{2} g^{\alpha \beta}(p)\left(\frac{\partial g_{\beta, \nu}(p)}{\partial x^{\mu}}+\frac{\partial g_{\mu, \beta}(p)}{\partial x^{v}}-\frac{\partial g_{\mu, \nu}(p)}{\partial x^{\beta}}\right)=0 .
$$

The explicit form of the covariant derivative is a consequence of this result and it is equal to the ordinary derivative in flat spacetime:

$$
\nabla_{\mu} v^{v}=\partial_{\mu} v^{v}+H_{\mu \alpha}^{v} v^{\alpha}=\partial_{\mu} v^{v}
$$

Now it is possible to define the spinorial connection using the covariant derivative:

$$
\omega_{\mu a b}=e_{a}^{v} \nabla_{\mu} e_{b v}=e_{a}^{v} \partial_{\mu} e_{b v} .
$$

It is trivial to demonstrate that all the connection coefficients vanish since the vierbein depends on the momentum but not on the coordinates. Finally, we obtain the total geometric covariant derivative, which will be useful in defining the minimal extension of the Standard Model (SM) of particle physics:

$$
D_{\mu} v_{a}^{v}=\partial_{\mu} v_{a}^{v}+\Gamma_{\mu \alpha}^{v} v_{a}^{\alpha}-\omega_{\mu v}^{a} v_{b}^{v} \simeq \partial_{\mu} v_{b}^{v} .
$$

The resulting spacetime geometry is therefore a flat Finsler pseudo-structure [43-48], whereas the associated momentum space is asymptotically flat.

\subsection{Modified Poincaré Brackets}

As a final result about the geometrical structure introduced by HMSR, we report the modified Poincaré brackets, computed for the local structure using the vierbein projectors:

$$
\begin{aligned}
\left\{\widetilde{x}^{\mu}, \widetilde{x}^{v}\right\} & =\left\{x^{i} e_{i}^{\mu}(p), x^{j} e_{j}^{v}(p)\right\}=\left\{x^{i}, e_{j}^{v}(p)\right\} e_{i}^{\mu}(p) x^{j}+\left\{e_{i}^{\mu}(p), x^{j}\right\} x^{i} e_{j}^{v}(p) \\
\left\{\widetilde{x}^{\mu}, \widetilde{p}_{v}\right\} & =\left\{x^{i} e_{i}^{\mu}(p), p_{j} e_{v}^{j}(p)\right\}=\left\{x^{i}, p_{j}\right\} e_{i}^{\mu}(p) e_{v}^{j}(p)+\left\{x^{i}, e_{v}^{j}(p)\right\} e_{i}^{\mu}(p) p_{j} \\
& =\delta_{v}^{\mu}+\left\{x^{i}, e_{v}^{j}(p)\right\} e_{i}^{\mu}(p) p_{j} \\
\left\{\widetilde{p}_{\mu}, \widetilde{p}_{v}\right\} & =\left\{p_{i} e_{\mu}^{i}(p), p_{j} e_{v}^{j}(p)\right\}=0,
\end{aligned}
$$

where $\left\{\widetilde{x}^{\mu}\right\}$ are the local coordinates and $\left\{x^{i}, e_{v}^{j}(p)\right\} \neq 0$ since the vierbein $e_{v}^{j}(p)$ is a function of the momentum $p_{\mu}$. Hence, time and space coordinates do not commute anymore [31]. Using the explicit form of the vierbein Equation (39) and admitting for the perturbation functions the approximations (See Appendix A) :

$$
\begin{aligned}
& h\left(\frac{|\vec{p}|}{E}\right)=\alpha\left(\frac{|\vec{p}|}{E}\right)+\ldots \\
& k\left(\frac{|\vec{p}|}{E}\right)=\beta\left(\frac{|\vec{p}|}{E}\right)+\ldots
\end{aligned}
$$


it is possible to obtain the following relations valid at the first perturbative order:

$$
\begin{aligned}
& {\left[\widetilde{x}^{\mu}, \widetilde{x}^{v}\right]=\theta^{\mu v}} \\
& {\left[\widetilde{x}^{\mu}, \widetilde{p}_{v}\right]=\delta_{v}^{\mu}+\left[x^{i}, e_{v}^{j}(p)\right] e_{i}^{\mu}(p) p_{j} \simeq \delta_{v}^{\mu}\left(1-\frac{1}{2}(\alpha+3 \beta)\left(\frac{|\vec{p}|}{E}\right)^{2}\right)} \\
& {\left[\widetilde{p}_{\mu}, \widetilde{p}_{v}\right]=0,}
\end{aligned}
$$

with the antisymmetric matrix $\theta^{\mu v}$ that satisfies the relations:

$$
\begin{aligned}
& \theta^{i j}=\theta^{00}=0 \quad \forall i, j \in 1,2,3 \\
& \theta^{0 i}=-\theta^{i 0} \simeq \frac{1}{2}\left(\beta\left(\frac{|\vec{p}| x^{i}}{E^{2}}\right)-\alpha\left(\frac{|\vec{p}| x^{0}}{E|\vec{p}|}\right)\right) \simeq \frac{1}{2}(\beta-\alpha)
\end{aligned}
$$

in the high energy limit, proving that the spacetime coordinates do not commute anymore [49] if the parameters $\alpha$ and $\beta$ are different, that is $h \neq k$ Equation (1).

The relation existing between noncommutative field theory and LIV was analyzed in [50]; here we introduced a framework to investigate this point in the context of modified covariance.

\section{Minimal Extension of the Standard Model in Covariance-Preserving Scenario}

In the HMSR framework, the SM of particle physics can be amended in order to include the QG-caused perturbations preserving the covariance of the theory, even if in an amended formulation. Following, for instance, a strategy analogous to that used for the isotropic sector of the Standard Model Extension (SME) [19], the theory formulation requires the definition of the modified Dirac matrices with the related Clifford algebra and spinors.

\subsection{Modified Clifford Algebra and Spinors}

The Dirac matrices acquire an explicit dependence on the particle momentum and again using the vierbein projectors Equation (39) they can be written as:

$$
\Gamma^{\mu}=e_{a}^{\mu}(p) \gamma^{a} \quad \Gamma_{5}=\frac{\epsilon^{\mu v \alpha \beta}}{4 !} \Gamma_{\mu} \Gamma_{\nu} \Gamma_{\alpha} \Gamma_{\beta}=\gamma_{5} .
$$

The $\gamma_{5}$ matrix is constant and this means that the chiral projectors are not affected.

The Dirac matrices modified via Equation (51) satisfy the defining relation of the Clifford algebra:

$$
\left\{\Gamma_{\mu}, \Gamma_{v}\right\}=2 g_{\mu v}(p)=2 e_{\mu}^{a}(p) \eta_{a b} e_{v}^{b}(p) .
$$

The definition of spinor fields is now amended preserving the usual plane-wave formulation:

$$
\begin{aligned}
& \psi^{+}(x)=u_{r}(p) \mathrm{e}^{-\mathrm{i} p p_{\mu} x^{\mu}} \\
& \psi^{-}(x)=v_{r}(p) \mathrm{e}^{\mathrm{i} p_{\mu} x^{\mu}} .
\end{aligned}
$$

The normalization of the spinors $u_{r}(p)$ and $v_{r}(p)$ is modified since these are defined using the newly introduced metric Equation (22) and the related internal product.

From the previous definitions the modified Dirac equation can be derived:

$$
\left(i \Gamma^{\mu} \partial_{\mu}-m\right) \psi=0 .
$$

An important consistency check of the new formalism can be obtained verifying that Equation (54) implies the MDR Equation (1). 


$$
\begin{aligned}
& \left(i \Gamma^{\mu} \partial_{\mu}+m\right)\left(i \Gamma^{\mu} \partial_{\mu}-m\right) \psi^{+}=0 \\
& \Rightarrow\left(\Gamma^{\mu} p_{\mu}+m\right)\left(\Gamma^{\mu} p_{\mu}-m\right) u_{r}(p)=0 \\
& \Rightarrow\left(\frac{1}{2}\left\{\Gamma^{\mu}, \Gamma^{\nu}\right\} p_{\mu} p_{v}-m^{2}\right) u_{r}(p)=0 \\
& \Rightarrow\left(p_{\mu} g^{\mu v} p_{v}-m^{2}\right) u_{r}(p)=0 .
\end{aligned}
$$

As a final result, a minimal extension of the SM can be obtained from the formalism here introduced. Indeed using the vierbein elements to project to a common Minkowski support spacetime the physical quantities related to different interacting particles and using the explicit form of the total covariant derivative Equation (45), the minimal extension of the SM can be formulated for a flat spacetime. Here we illustrate the amended formulation of quantum electrodynamics (QED), whose Lagrangian can be written in the form:

$$
\mathcal{L}=\sqrt{|\operatorname{det}[g]|} \bar{\psi}\left(i \Gamma^{\mu} \partial_{\mu}-m\right) \psi+e \sqrt{|\operatorname{det}[\widetilde{g}]|} \bar{\psi} \Gamma_{\mu}\left(p, p^{\prime}\right) \psi \bar{e}_{a}^{\mu} A^{a},
$$

where the term $\sqrt{|\operatorname{det}[g]|}$ is borrowed from the formulation of QFT in curved spacetime. The vierbein element $\bar{e}$ is related to the gauge field $A^{a}(x)$. The gauge field is supposed as Lorentz-covariant in the usual meaning, that is, the MAV of photons is the usual speed of light $c$. The gauge field therefore is set on a Minkowski spacetime $\left(T M, \eta_{a b}\right)$ and the vierbein is given by: $\bar{e}_{a}^{\mu}=\delta_{a}^{\mu}$. The QG corrections can be introduced in the generic gauge boson sector modifying the definition of the vierbein related to the gauge field, as done for the massive fermion fields.

The interaction is governed by the conserved current.

$$
J_{\mu}=e \sqrt{\left|\operatorname{det} \frac{1}{2}\left\{\Gamma_{\mu}, \Gamma_{v}\right\}\right|} \bar{\psi} \Gamma_{\mu} \psi=e \sqrt{|\operatorname{det}[g]|} \bar{\psi} \Gamma_{\mu} \psi .
$$

In the low-energy scenario, the covariance perturbations in the conserved current are negligible, whereas in the high-energy limit the formulation admits a constant form, since one can consider the incoming and outgoing momenta with the same constant high-energy limit.

\subsection{Gauge Symmetry}

The SM minimal extension obtained in the context of HMSR preserves the classic internal gauge symmetry $S U(3) \otimes S U(2) \otimes U(1)$. This result can be stated formulating an amended version of the Coleman-Mandula theorem [26]. The modified gauge symmetry group acquires the explicit form:

$$
\mathcal{P}(\{p\}) \otimes G_{\text {int }},
$$

where $\mathcal{P}(\{p\})$ is the kinematical symmetry group and is given by the direct product of the momentum-dependent Poincaré groups associated with the different particle species.

$$
\mathcal{P}(\{p\})=\bigotimes_{i} \mathcal{P}^{(i)}\left(p_{(i)}\right)
$$

and $G_{\text {int }}$ is the internal gauge symmetry group, in this case the usual SM gauge group:

$$
G_{\text {int }}=S U(3) \otimes S U(2) \otimes U(1) .
$$




\subsection{Modified Kinematics}

HMSR theory perturbs the kinematics geometrizing the interaction of free propagating particles with the supposed quantized structure of the spacetime. In this work, we investigate the phenomenological effects introduced by QG in the UHECRs propagation, where the main effects are caused by the modification of the kinematics. The detectable effects are caused by the interaction of different particle species, which modify in a proper way the related spacetime.

Introducing a generalized internal product in the momentum space for more than two different interacting particle species, it is possible to obtain a generalized formulation for the Mandelstam variables $s, t$ and $u$, which are the dynamical invariant quantities linked to a reaction.

Considering $p$ and $q$ as the momenta of two interacting particles of different species, the internal product of their sum can be defined as:

$$
(p+q \mid p+q)=\left(p_{\mu} e_{a}^{\mu}(p)+q_{\mu} \tilde{e}_{a}^{\mu}(q)\right) \eta^{a b}\left(p_{\nu} e_{b}^{v}(p)+q_{v} \tilde{e}_{b}^{v}(q)\right),
$$

where the vierbeins $e_{a}^{\mu}(p)$ and $\tilde{e}_{a}^{\mu}(q)$ are associated, respectively, with the two different particle species. The formulation of the modified internal product can be simplified in the form:

$$
\begin{aligned}
& (p+q \mid p+q)=\left(\begin{array}{l}
p \\
q
\end{array}\right)^{t} \cdot G \cdot\left(\begin{array}{l}
p \\
q
\end{array}\right)=\left(\begin{array}{ll}
p & q
\end{array}\right)\left(\begin{array}{cc}
g^{\mu v}(p) & e^{a \mu}(p) \tilde{e}_{a}^{\beta}(q) \\
\tilde{e}^{a \alpha}(q) e_{a}^{v}(p) & \tilde{g}^{\alpha \beta}(q)
\end{array}\right)\left(\begin{array}{l}
p \\
q
\end{array}\right) \\
& =p_{\mu} g^{\mu v}(p) p_{v}+p_{\mu} e^{a \mu} \tilde{e}_{a}^{\beta}(q) q_{\beta} \\
& +q_{\alpha} \tilde{e}^{a \alpha}(q) e_{a}^{v}(p) p_{v}+q_{\alpha} \tilde{g}^{\alpha \beta}(q) q_{\beta},
\end{aligned}
$$

using the generalized metric:

$$
G=\left(\begin{array}{cc}
g^{\mu \nu}(p) & e^{a \mu}(p) \tilde{e}_{a}^{\beta}(q) \\
\tilde{e}^{a \alpha}(q) e_{a}^{v}(p) & \tilde{g}^{\alpha \beta}(q)
\end{array}\right)
$$

The inner product defined in Equation (62) is invariant with respect to the Modified Lorentz Transformations (MLT) introduced in HMSR:

$$
\Lambda=\left(\begin{array}{cc}
\Lambda_{\mu}^{\mu^{\prime}} & 0 \\
0 & \tilde{\Lambda}_{\alpha}^{\alpha^{\prime}}
\end{array}\right) .
$$

The inner product remains invariant under the action of such generalized Lorentz transformations, indeed one can obtain:

$$
\begin{aligned}
(p+q \mid p+q) & =\left(\begin{array}{l}
p \\
q
\end{array}\right)^{t} \cdot G \cdot\left(\begin{array}{l}
p \\
q
\end{array}\right)=\left(\Lambda\left(\begin{array}{l}
p \\
q
\end{array}\right)\right)^{t} \cdot \Lambda \cdot G \cdot \Lambda^{t} \cdot \Lambda\left(\begin{array}{l}
p \\
q
\end{array}\right) \\
& =(\Lambda(p+q) \mid \Lambda(p+q)) .
\end{aligned}
$$

$\Lambda \cdot G \cdot \Lambda^{t}$ is the metric evaluated for the two particle momenta $\Lambda p, \tilde{\Lambda} q$.

The new formalism here introduced guarantees that HMSR theory can deal with the interaction of different particle species in the context of a QG-modified kinematics without the necessity of the introduction of a preferred reference frame.

\section{Ultra-High-Energy Cosmic Ray Propagation}

Before investigating the QG-induced phenomenology in UHECRs physics, it is useful to illustrate the standard physics predictions for these highly energetic particles. During their propagation, at sufficiently high energies, UHECRs can interact with the CMB and depending on their nature and energy their flux is attenuated. For instance, a propagating 
CR bare nucleus $X$ with atomic number $A$ can undergo a photo-dissociation process interacting with the $\mathrm{CMB}$ and emitting one or more nucleons:

$$
X_{A}+\gamma \rightarrow X_{(A-k)}+n_{1}+\ldots+n_{k},
$$

where $n_{i}$ is a generic nucleon. The proton propagation is influenced by different interaction processes with the $\mathrm{CMB}$, hence it can undergo, for instance, a pair production process:

$$
p+\gamma \rightarrow p+e^{-}+e^{+} .
$$

This process is the main interaction mechanism with the CMB for CR protons with an energy lower than the threshold $E \sim 5 \times 10^{19} \mathrm{eV}$. The dominant process for UHECR protons with an energy exceeding this threshold is the $\Delta$ particle resonance photopion production process:

$$
\begin{aligned}
& p+\gamma \rightarrow \Delta \rightarrow p+\pi^{0} \\
& p+\gamma \rightarrow \Delta \rightarrow n+\pi^{+} .
\end{aligned}
$$

These dissipation mechanisms make the universe opaque to the propagation of $\mathrm{CR}$, particularly for the most energetic component (UHECR), with an energy that exceeds the threshold $E \sim 5 \times 10^{19} \mathrm{eV}$. In this work, we are particularly interested in the UHECRs propagation since $C R$ s can be useful in investigating $Q G$ phenomenology thanks to their huge energy and propagation length. QG effects in some theories are expected as more evident at high energies and their perturbations can sum up during the propagation of this kind of particles. The photopion production is the main attenuation interaction of UHECRs and is the core mechanism for the so-called GZK cut-off phenomenon for protons [2,3]. This effect poses an upper limit on the energy of protons detected at ground and coming from distant sources. Since through this effect a particle dissipates energy, but is not annihilated, a proton with enough energy can undergo the same interaction process again, and can undergo what is known as a stochastic dissipation process. This way, it becomes possible to evaluate the attenuation length, defined as the average distance that the proton has to travel in order to reduce its energy by a factor of $1 / \mathrm{e}$. The inverse of the attenuation length is given by [51]:

$$
\begin{aligned}
\frac{1}{l_{p \gamma}} & =\int_{\epsilon_{\mathrm{th}}}^{+\infty} n(\epsilon) \mathrm{d} \epsilon \int_{-1}^{+1} \frac{1}{2} s(1-\mu) \sigma_{p \gamma}(s) K(s) \mathrm{d} \mu \\
& =\int_{\epsilon_{\mathrm{th}}}^{+\infty} n(\epsilon) \mathrm{d} \epsilon \int_{-1}^{+1} \frac{1}{2} s\left(1-v_{p} \cos \theta\right) \sigma_{p \gamma}(s) K(s) \mathrm{d} \cos \theta,
\end{aligned}
$$

where $\sigma_{p \gamma}(s)$ is the proton-photon interaction cross section as a function of the squared center of mass energy (the Mandelstam $s$ variable), $n(\epsilon)$ represents the background photon density per unit volume and photon energy $\epsilon, \mu=\cos \theta$ is the impact parameter and $\epsilon_{\text {th }}$ is the interaction threshold energy. $K(s)$ represents the reaction inelasticity, that is, the energy fraction available for secondary-particle production during the reaction. Complementary to the inelasticity is the elasticity function, defined as the energy fraction preserved by the primary particle after the interaction, $\eta=E_{\text {out }} / E_{\text {in }}$ with the incoming particle energy $E_{\text {in }}$ and the residual energy $E_{\text {out }}$. Elasticity and inelasticity are connected by the simple relation: $K=(1-\eta)$.

The Mandelstam $s$ can be computed introducing the photon four-momentum $\left(\epsilon^{\prime}, \vec{p}_{\gamma}^{\prime}\right)$ defined in the rest frame of the nucleus. In the high-energy limit approximation for the proton velocity $v_{p} \simeq 1$, with $\mathrm{d} s=-2 E_{p} \in \mathrm{d} \cos \theta$, the following relations hold:

$$
\begin{aligned}
& s=\left(m_{p}+\epsilon^{\prime}\right)^{2}-\left|\vec{p}_{\gamma}^{\prime}\right|^{2}=m_{p}^{2}+2 m_{p} \epsilon^{\prime} \\
& \epsilon^{\prime}=\gamma \epsilon\left(1-v_{p} \cos \theta\right),
\end{aligned}
$$


hence, the inverse of the attenuation length Equation (69) can be written as:

$$
\frac{1}{l_{p \gamma}}=\frac{1}{2 \gamma^{2}} \int_{\epsilon_{\mathrm{th}}^{\prime} / 2 \gamma}^{+\infty} \mathrm{d} \epsilon \frac{n(\epsilon)}{\epsilon^{2}} \int_{\epsilon_{\mathrm{th}}^{\prime}}^{\epsilon_{\max }^{\prime}=2 \gamma \epsilon} \frac{1}{2} \epsilon^{\prime} \sigma_{p \gamma}\left(\epsilon^{\prime}\right) K\left(\epsilon^{\prime}\right) \mathrm{d} \epsilon^{\prime},
$$

where the primed quantities are defined in the proton rest frame, whereas the other quantities are defined in the laboratory rest frame. Since the $n(\epsilon)$ distribution is a Planckian function of the temperature $T$, a further simplification of the previous relation of Equation (73) is possible, obtaining the explicit form for the inverse of the attenuation length [5]:

$$
\frac{1}{l_{p \gamma}}=-\frac{k_{\mathrm{B}} T}{2 \pi^{2} \gamma^{2}} \int_{\epsilon_{\mathrm{th}}^{\prime}}^{+\infty} \epsilon^{\prime} \sigma_{p \gamma}\left(\epsilon^{\prime}\right) K\left(\epsilon^{\prime}\right) \ln \left(1-e^{-\epsilon^{\prime} / 2 K T \gamma}\right) \mathrm{d} \epsilon^{\prime} .
$$

The inelasticity computed for the standard physics case is given by the relation [51]:

$$
K(s)=\frac{1}{2}\left(1-\frac{m_{p}^{2}-m_{\pi}^{2}}{s}\right) .
$$

\section{QG Introduced Phenomenology in UHECR Propagation}

In this work, we present a review of the QG presumed effects in the UHECR sector as predicted in the context of the HMSR model [26]. The results, already presented in a previous work [8], are better justified by a generalized formulation of the model, that indicates a strategy to set the investigation in curved spacetime. Moreover, in this work we conduct our analysis in the HMSR framework, but in the context of a Finsler geometry structure obtained by starting from a MDR written in a more general form Equation (1) than the one given by Equation (3) and used in previous works $[7,8]$.

Following the theoretical framework introduced by HMSR, the kinematics of free particles are modified in order to include the QG effects. The kinematical perturbations modify the allowed phase space for the reaction and may therefore influence the processes involved in UHECR propagation. The introduction of QG phenomenology can indeed affect the photopion production mechanism, the core reaction underlying the GZK phenomenon [5-8]. The QG-caused reduction of the allowed phase space can modify the inelasticity $K$ Equation (75). The consequent reduction of the inelasticity means that the incoming UHECR proton dissipates less energy during the GZK process and therefore the resulting opacity horizon is enlarged with respect to the standard model physics prediction.

\subsection{Constraints from $\Delta$ Resonance Creation}

The photopion production requires a $\Delta$ particle creation (Equation (68)) and can occur passing through a real $\Delta$ particle, in the case of the dominant process, or alternatively through a virtual one. The introduction of QG phenomenology can presumably modify only the GZK cut-off phenomenon, enlarging the foreseen opacity sphere without a complete suppression. The observed UHECR flux suppression can be caused by the exhaustion of the sources energy or by the Universe opacity to the propagation of CR $[52,53]$. In the last scenario, an uncertainty exists about the dimension of the foreseen opacity horizon. The production of the real $\Delta$ particle must therefore be preserved in order to foresee only small QG-induced deviations from the standard physics predictions for the GZK process. The fourmomenta used in the following computations are considered as covariant vectors in order to simplify the use of the MLT for changing the reference frame. In the proton-CMB interaction process the production of a $\Delta$ particle is allowed if the proton-CMB interaction free energy, that is the Mandelstam $\sqrt{s}$ variable, exceeds the rest energy of the particle resonance. As a consequence of this threshold energy consideration, a first constraint on the magnitude of the QG perturbation parameters can be posed. Using the generalized internal product and the formalism developed for the modified kinematics in the Equations (61) and (62) one can 
write the Mandelstam $s$ variable as a function of the proton and the photon four-momenta, respectively, $p_{(p)}=\left(E_{(p)}, \vec{p}_{(p)}\right)$ and $p_{(\gamma)}=\left(E_{(\gamma)}, \vec{p}_{(\gamma)}\right)$ :

$$
\begin{aligned}
s= & \left(p_{(p)}^{\mu} e_{\mu}^{a}\left(p_{p}\right)+p_{\gamma}^{\mu} e_{\mu}^{a}\left(p_{\gamma}\right)\right) \eta_{a b}\left(p_{(p)}^{v} e_{v}^{b}\left(p_{p}\right)+p_{\gamma}^{v} e_{v}^{b}\left(p_{\gamma}\right)\right) \\
= & p_{(p)}^{\mu} e_{v}^{a}\left(p_{p}\right) \eta_{a b} e_{v}^{b}\left(p_{p}\right) p_{(p)}^{v}+p_{(p)}^{\mu} e_{v}^{a}\left(p_{p}\right) \eta_{a b} e_{v}^{b}\left(p_{\gamma}\right) p_{(\gamma)}^{v} \\
& +p_{(\gamma)}^{\mu} e_{v}^{a}\left(p_{\gamma}\right) \eta_{a b} e_{v}^{b}\left(p_{p}\right) p_{(p)}^{v} \\
= & p_{(p)}^{\mu} g_{\mu v}\left(p_{p}\right) p_{(p)}^{v}+2 p_{(p)}^{\mu} e_{\mu}^{a}\left(p_{p}\right) \eta_{a b} \delta_{v}^{b} p_{(\gamma)}^{v} \geq m_{\Delta}^{2} .
\end{aligned}
$$

The vierbein Equation (39) has been used to project the momenta of different particles to the common support Minkowski spacetime and the symmetry of the mixed product of $p_{p}$ and $p_{\gamma}$ has been used. Photons are assumed to be Lorentz-invariant, hence the related vierbein is defined as usual: $e_{\mu}^{a}\left(p_{\gamma}\right)=\delta_{\mu}^{a}$. Using the explicit form of the squared Finsler norm $F^{2}(p)$ given in Equation (35), the following inequality can be derived reordering the terms of the previous relation in an opportune manner:

$$
\begin{aligned}
& E_{p}^{2}\left(1-h_{p}\left(p_{p}\right)\right)-\left|\vec{p}_{p}\right|^{2}\left(1-k_{p}\left(p_{p}\right)\right)+2 E_{p} E_{\gamma}\left(1-\frac{1}{2} h_{p}\left(p_{p}\right)\right) \\
& -2 \vec{p}_{p} \cdot \vec{p}_{\gamma}\left(1-\frac{1}{2} k_{p}\left(p_{p}\right)\right) \geq m_{\Delta}^{2},
\end{aligned}
$$

where $h_{p}\left(p_{p}\right)$ and $k_{p}\left(p_{p}\right)$ are the proton QG perturbation functions taken from the MDR Equation (39). The following inequality can be derived from the previous Equation (77) using the MDR Equation (1):

$$
2\left(h_{p}\left(p_{p}\right)-k_{p}\left(p_{p}\right)\right) E_{p}^{2}-E_{p} E_{\gamma}(4+\underbrace{\left(h_{p}\left(p_{p}\right)-k_{p}\left(p_{p}\right)\right)}_{O(1)})+m_{\Delta}^{2}-m_{p}^{2} \leq 0,
$$

where $m_{\Delta} \simeq 1232 \mathrm{MeV}, m_{p} \simeq 938 \mathrm{MeV}$. The covariant formulation of the model is fundamental since it allows the change of the reference frame. Indeed, in order to simplify the computation, it is possible to choose the more suitable reference frame, where the involved energy scales ratio allows the suppression of some perturbative terms. One can therefore consider the laboratory frame, where the proton energy is much higher than the CMB one. Considering that the UHECR proton energy has an upper limit $E_{p} \lesssim 10^{21} \mathrm{eV}[1]$ and taking into account the tiny average value of the $\mathrm{CMB}$ energy $E_{\gamma} \simeq 1.16 \times 10^{-3} \mathrm{eV}$, one can neglect the $O(1)$ contributions in the second term obtaining:

$$
2\left(h_{p}\left(p_{p}\right)-k_{p}\left(p_{p}\right)\right) E_{p}^{2}-4 E_{p} E_{\gamma}+m_{\Delta}^{2}-m_{p}^{2} \leq 0 .
$$

Defining the function $f_{p}=h_{p}-k_{p}$ the result obtained is comparable with the one presented in $[7,8]$. The derived inequality must be satisfied in order to produce a $\Delta$ resonance, otherwise the QG perturbations totally suppress the GZK effect. Imposing the validity of the previous relation the following constraint can be derived:

$$
f_{p}\left(p_{p}\right) \leq \frac{2 E_{\gamma}}{m_{\Delta}^{2}-m_{p}^{2}}
$$

Substituting the approximated average value of the CMB energy in the previous inequality one can obtain the approximated constraint:

$$
f_{p}\left(p_{p}\right) \lesssim 10^{-21}
$$

where $m_{\Delta}^{2}-m_{p}^{2} \sim 10^{18} \mathrm{eV}$ and $E_{\gamma} \sim 10^{-3} \mathrm{eV}$. The obtained constraint is consistent with the upper limit $4.5 \cdot 10^{-23}$ obtained numerically in [5] for the QG perturbation magnitude. 
As a final remark from the relations Equations (78) and (80), it follows that in order to generate visible effects on the GZK phenomenon, the QG perturbation function $f_{p}$ must satisfy the relation:

$$
f_{p}\left(p_{p}\right)=h_{p}\left(p_{p}\right)-k_{p}\left(p_{p}\right)>0 .
$$

Indeed from Equation (78) the high-energy limit excludes the possibility of any suppression of the GZK cut-off in the case of a negative function $f_{p}$. We underline that the choice of positive values of the perturbation $f_{p}$ corresponds to the introduction of an effective MAV in the MDR of Equation (1) for every massive particle, which is lower than the standard speed of light.

\subsection{Modified Inelasticity}

The introduction of QG perturbations can modify the phase space allowed for the photopion production process, determining a modification of the inelasticity function $K(s)$ Equation (75). In this work, we evaluate the new inelasticity in the context of the generalized Finsler geometry here introduced following the approach of $[7,8]$, originally inspired by $[5,6]$. In the following, all the computations are conducted again considering the four-momenta as covariant vectors, in order to simplify the computations transforming the reference frame with the MLT. The introduction of the center-of-mass (CM) reference frame is useful and it is defined via the relation:

$$
\vec{p}_{p}^{*}+\vec{p}_{\pi}^{*}=0
$$

where the momenta are defined on the common Minkowski support spacetime $\left(T M, \eta_{a b}\right)$ and $*$ labels the quantities related to the CM frame.

The next element necessary in evaluating the change from the CM to a generic reference frame is the $\gamma_{\mathrm{CM}}$ Lorentz factor. Starting from the free energy available for the photopion production $\sqrt{s}=E_{p}^{*}+E_{\pi}^{*}$ one can obtain the relation:

$$
\gamma_{\mathrm{CM}}\left(E_{p}^{*}+E_{\pi}^{*}\right)=\gamma_{\mathrm{CM}} \sqrt{s}=\left(E_{p}+E_{\pi}\right) \Rightarrow \gamma_{\mathrm{CM}}=\frac{E_{p}+E_{\pi}}{\sqrt{s}}=\frac{E_{\mathrm{tot}}}{\sqrt{s}} .
$$

Using the CM definition Equation (83) the relation $\left|\vec{p}_{p}^{*}\right|=\left|\vec{p}_{\pi}^{*}\right|$ follows and the fourmomentum of the photopion can be written in the CM reference frame as $p_{\pi}^{*}=(\sqrt{s}-$ $\left.E_{p}^{*}, \vec{p}_{p}^{*}\right)=\left(E_{\pi}^{*}, \vec{p}_{\pi}^{*}\right)$. From the latter, the free energy necessary to produce a photopion in the CM frame can be computed using the squared Finsler norm Equation (35):

$$
\begin{aligned}
& p_{(\pi)}^{\mu} e_{\mu}^{a}\left(p_{\pi}\right) \eta_{a b} e_{v}^{b}\left(p_{\pi}\right) p_{(\pi)}^{v}=m_{\pi}^{2} \\
\Rightarrow & \left(\sqrt{s}-E_{(p)}^{*}\right)^{2}\left(1-h_{\pi}\left(p_{\pi}\right)\right)-\left|\vec{p}_{(p)}^{*}\right|\left(1-k_{\pi}\left(p_{\pi}\right)\right) \\
= & \left(s-2 \sqrt{s} E_{(p)}^{*}\right)\left(1-h_{\pi}\left(p_{\pi}\right)\right)+E_{(p)}^{* 2}\left(1-h_{p}\left(p_{p}\right)\right)+E_{(p)}^{* 2}\left(h_{p}\left(p_{p}\right)-h_{\pi}\left(p_{\pi}\right)\right) \\
& \quad-\left|\vec{p}_{(p)}^{*}\right|^{2}\left(1-k_{p}\left(p_{p}\right)\right)-\left|\vec{p}_{(p)}^{*}\right|^{2}\left(k_{p}\left(p_{p}\right)-k_{\pi}\left(p_{\pi}\right)\right) \\
= & \left(s-2 \sqrt{s} E_{(p)}^{*}\right)\left(1-h_{\pi}\left(p_{\pi}\right)\right)+m_{p}^{2}\left(1-h_{\pi}\left(p_{\pi}\right)\right)+E_{(p)}^{* 2}\left(h_{p}\left(p_{p}\right)-h_{\pi}\left(p_{\pi}\right)\right) \\
& \quad-\left|\vec{p}_{(p)}^{*}\right|^{2}\left(k_{p}\left(p_{p}\right)-k_{\pi}\left(p_{\pi}\right)\right)=m_{\pi}^{2},
\end{aligned}
$$

$h_{p}$ and $k_{p}$ are the perturbation functions of the proton MDR of Equation (1) and $h_{\pi}$ and $k_{\pi}$ are the analogous functions for the pion MDR, $p$ and $\pi$ label, respectively, the elements related to the proton and the pion.

We underline that the computation made here for the modifications induced on the inelasticity are still valid in the context of the more restrictive Finsler geometry. Indeed, the computation is conducted using the MDR Equation (1), that is the norm Equation (35). 
From the previous equation and neglecting the $O(2)$ perturbations in Equation (35), the following equality can be obtained:

$$
\begin{aligned}
E_{p}^{*} & =\frac{s+\left(m_{p}^{2}-m_{\pi}^{2}\right)\left(1+h_{\pi}\right)+E_{p}^{* 2}\left(h_{p}-h_{\pi}\right)-\left|\vec{p}_{p}^{*}\right|^{2}\left(k_{p}-k_{\pi}\right)}{2 \sqrt{s}} \\
& =\frac{s+\left(m_{p}^{2}-m_{\pi}^{2}\right)\left(1+h_{\pi}\right)+h_{p \pi} E_{p}^{* 2}-k_{p \pi}\left|\vec{p}_{p}^{*}\right|^{2}}{2 \sqrt{s}}=F(s),
\end{aligned}
$$

where $h_{p \pi}=h_{p}-h_{\pi}$ and $k_{p \pi}=k_{p}-k_{\pi}$ are the QG perturbation parameters. Considering the high-energy limit $E_{p}^{*} \simeq\left|\vec{p}_{p}^{*}\right|$ in the previous relation the residual proton energy after the photopion reaction can be increased if the QG parameters satisfy the inequality $h_{p \pi}>k_{p \pi}$ and therefore the relation $h_{p \pi}>0$. The proton-dominant correction $h_{p}$ must be larger than the pion one $k_{p}$ in order to produce a dilatation of the GZK sphere. In this work, we assume the gravitational nature of the perturbation effects caused by the supposed quantum structure of the background, hence the heavier particles have a bigger QG-induced modification. As a consequence, the correction factors of the pion can be considered negligible and it is possible to pose $h_{p \pi} \simeq h_{p}$ and $k_{p \pi} \simeq k_{p}$.

Now the following approximations can be introduced:

$$
\begin{aligned}
& p_{p}^{*} \simeq E_{p}^{*}=\left(1-K_{\pi}(\theta)\right) \sqrt{s} \\
& p_{\pi}^{*} \simeq E_{\pi}^{*}=K_{\pi}(\theta) \sqrt{s} .
\end{aligned}
$$

$E_{p}^{*}+E_{\pi}^{*}=\sqrt{s}$ represents the initial free total energy and $E_{p}^{\prime}$ and $E_{\pi}^{\prime}$ are the final energies of the proton and the pion, respectively.

Using the Lorentz transformations for changing reference frames, one can obtain the following:

$$
\begin{aligned}
& E_{p}^{\prime}=\gamma_{\mathrm{CM}}\left(E_{p}^{*}+\beta \cos \theta p_{p}^{*}\right) \\
& E_{p}^{*}=\left(1-k_{\pi}(\theta)\right) \sqrt{s},
\end{aligned}
$$

where $K_{\pi}(\theta)$ is the pion inelasticity depending on the impact angle.

In the high-energy limit, where for ultra-relativistic particles $|\vec{p}| \sim E$ and the velocity factor $\beta \sim 1$ and $\gamma_{\mathrm{CM}}$ is approximated by Equation (84), the following equation can be derived:

$$
\left(1-K_{\pi}(\theta)\right)=\frac{1}{\sqrt{s}}\left(F(s)+\cos \theta \sqrt{F(s)^{2}-m_{p}^{2}+2\left(h_{p}-k_{p}\right)|\vec{p}|^{2}}\right) .
$$

Posing $f_{p}=\left(h_{p}-k_{p}\right)$ in the previous relation, it is possible to obtain the following one:

$$
\left(1-K_{\pi}(\theta)\right)=\frac{1}{\sqrt{s}}\left(F(s)+\cos \theta \sqrt{F(s)^{2}-m_{p}^{2}+2 f_{p}|\vec{p}|^{2}}\right)
$$

where the latter result was obtained in $[7,8]$. The previous equation can be numerically solved in order to evaluate the inelasticity as a function of the collision angle $\theta$. The numerical computation is conducted in the high-energy limit, hence by using Equation (87) and $\sqrt{s}=E_{p}^{*}+E_{\pi}^{*}$ is the initial energy for the process.

The free energy $s$ can be written as:

$$
s=\left(E_{p}+\epsilon^{\prime}\right)^{2}-\left|\vec{p}_{\gamma}\right|^{2}=2 m_{p} \epsilon^{\prime}+m_{p}^{2},
$$

introducing the energy of the photon $\epsilon^{\prime}$ defined in the proton rest frame $\vec{p}_{p}=0$. 
The inelasticity is finally averaged over the interval $\theta \in[0, \pi]$ :

$$
K_{\pi}=\frac{1}{\pi} \int_{0}^{\pi} K_{\pi}(\theta) \mathrm{d} \theta .
$$

In the following, we plot in Figures 1 and 2 the inelasticity for different choices of the parameter $f_{p}=\left(h_{p}-k_{p}\right)$ in order to compare the standard physics predicted result with the QG induced modifications. The parameter $f_{p}$ is constrained to be positive in order to guarantee detectable QG effects. This hypothesis corresponds to the introduction of a MAV inferior to the classical speed of light $c$ for every massive particle species. The parameter is even constrained from above by the limit obtained in the previous section Equation (81), hence the plausible parameter must be included in the interval $0<f_{p} \lesssim 10^{-22}$. The inelasticity is plotted as a function of the proton energy $E_{p}$, defined in the laboratory reference frame, and the photon energy $\epsilon^{\prime}$, defined in the proton rest frame. Under these hypotheses, the QG perturbations cause a dramatic drop of the inelasticity value, which is more visible for increasing values of the parameter $f_{p}$. This implies a reduction of the allowed phase space for the photopion production process, and an enlargement of the foreseen proton opacity horizon for an increasing magnitude of the QG perturbation.

\section{Simulated Attenuation Length}

The impact of the QG perturbations on the UHECR free propagation can be evaluated computing the value of the attenuation length as a function of the modified inelasticity $K$ in Equation (74). In this work, we present the attenuation length, as in a previous work [8], obtained using an ad hoc modified version of the simulation software SimProp [27]. In this software version, we substituted the inelasticity predicted by the standard model physics with the modified one, being a function not only of the proton and the CMB energy but also of the QG parameter. The analysis is conducted neglecting the interaction of the UHE protons with the EBL. This interaction is dominant at energies below $\sim 5 \times 10^{19} \mathrm{eV}$. Slightly above this threshold the ELB interaction might give a $10 \%$ contribution in determining the attenuation length [54]. Hence the prediction presented in Figures 3 and 4 can be affected by ELB interaction at energies below the photopion production threshold $\left(\sim 5 \times 10^{19} \mathrm{eV}\right)$. For UHE protons exceeding this energy limit the effect produced seems to be a negligible reduction of the mean free path. In Figures 3 and 4 it is visible that the increase of the opacity horizon can be caused by the QG-induced modification of Lorentz covariance. In the QG-less scenario the attenuation length decreases for increasing CR energy values. In the presence of $Q G$, the interaction length at first decreases with the energy, but then after an inflection point, which depends on the energy, this quantity starts rising for increasing energy values. This effect is caused by the reduction of the average energy lost for every proton-CMB interaction caused by the reduction of the inelasticity. For a QG parameter of $f_{p} \sim 10^{-22}$ the modifications in the attenuation length are appreciable starting from an energy $E \sim 10^{20} \mathrm{eV}$ of the incoming proton, instead for a parameter $f_{p} \sim 2 \times 10^{-22}$ the perturbation starts being appreciable yet at an energy $E \sim 6 \times 10^{19} \mathrm{eV}$. The analysis can be improved taking into account even the electron-positron pair production. This effect is not dominant at the highest energies, but would presumably further increase the energy loss process. 

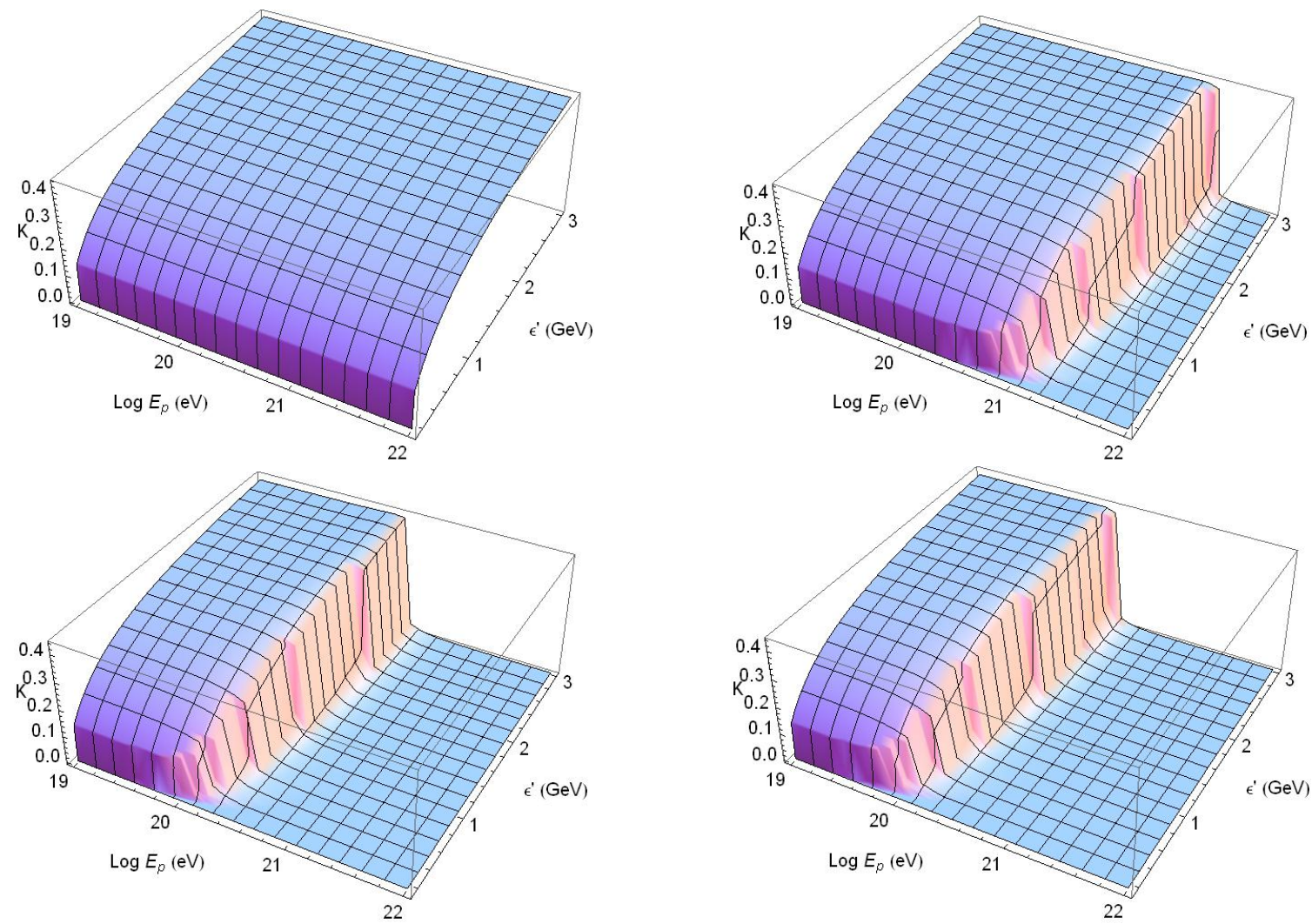

Figure 1. Comparison of standard physics inelasticity (LIV parameter $f_{p \pi} \simeq f_{p}=0$ ) with the modified ones obtained for different LIV parameters $\left(f_{p \pi} \simeq f_{p}=1 \times 10^{-24} ; 5 \times 10^{-23} ; 1 \times 10^{-22}\right)$ as a function of the proton energy $E_{p}$ and of the photon energy $\epsilon^{\prime}$ defined in the proton rest frame (3D plot).
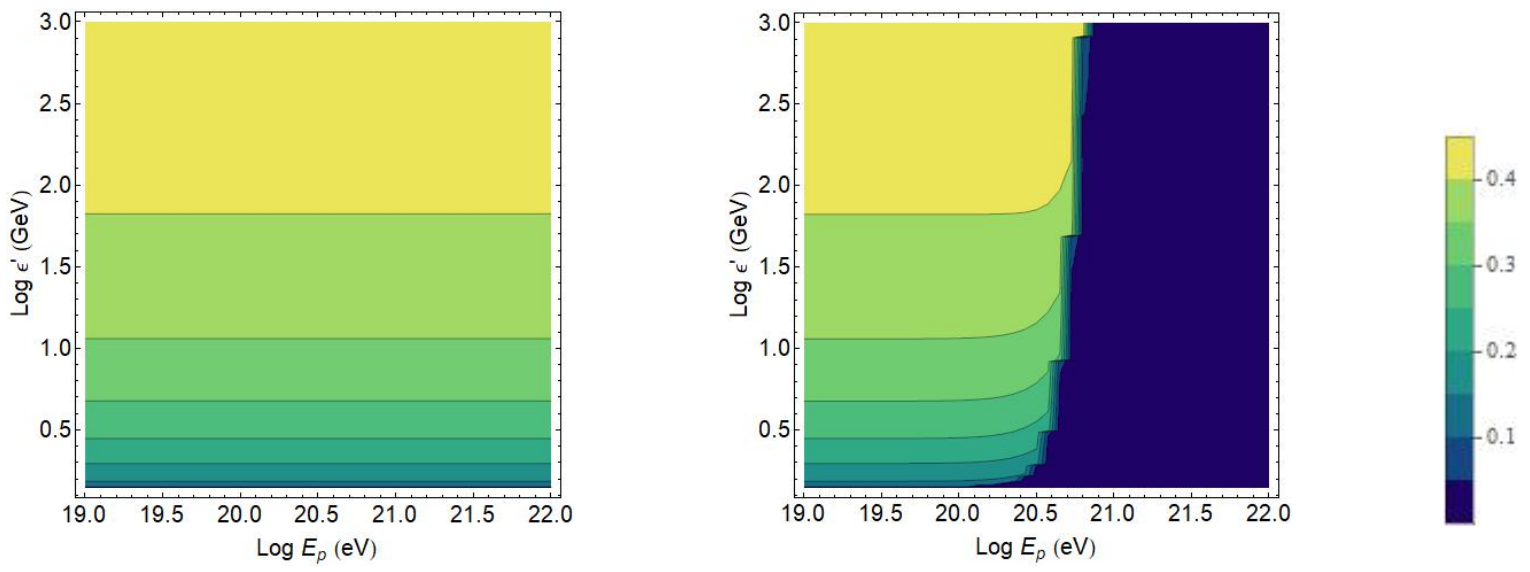

Figure 2. Cont. 

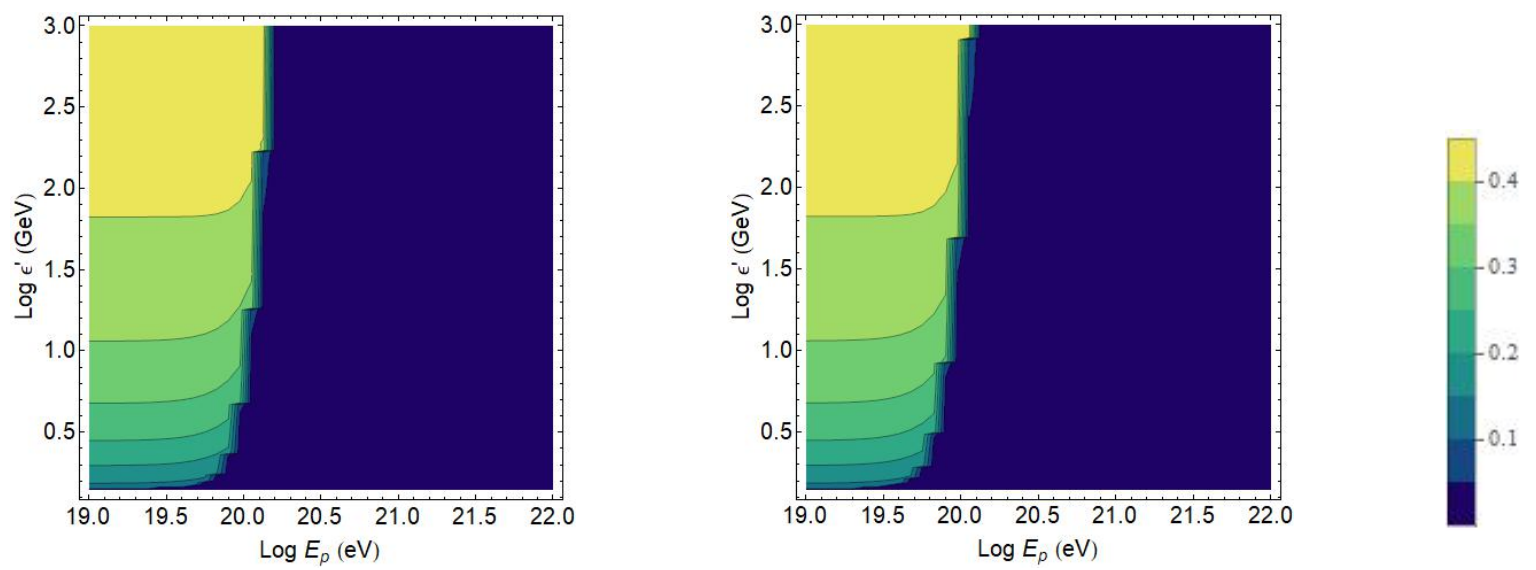

Figure 2. Level plot of the same comparison of the previous figure, the color scale indicates the numerical value of the inelasticity magnitude: standard case vs. the modified ones, obtained as a function of the proton energy $E_{p}$ and of the photon energy $\epsilon^{\prime}$ defined in the proton rest frame.

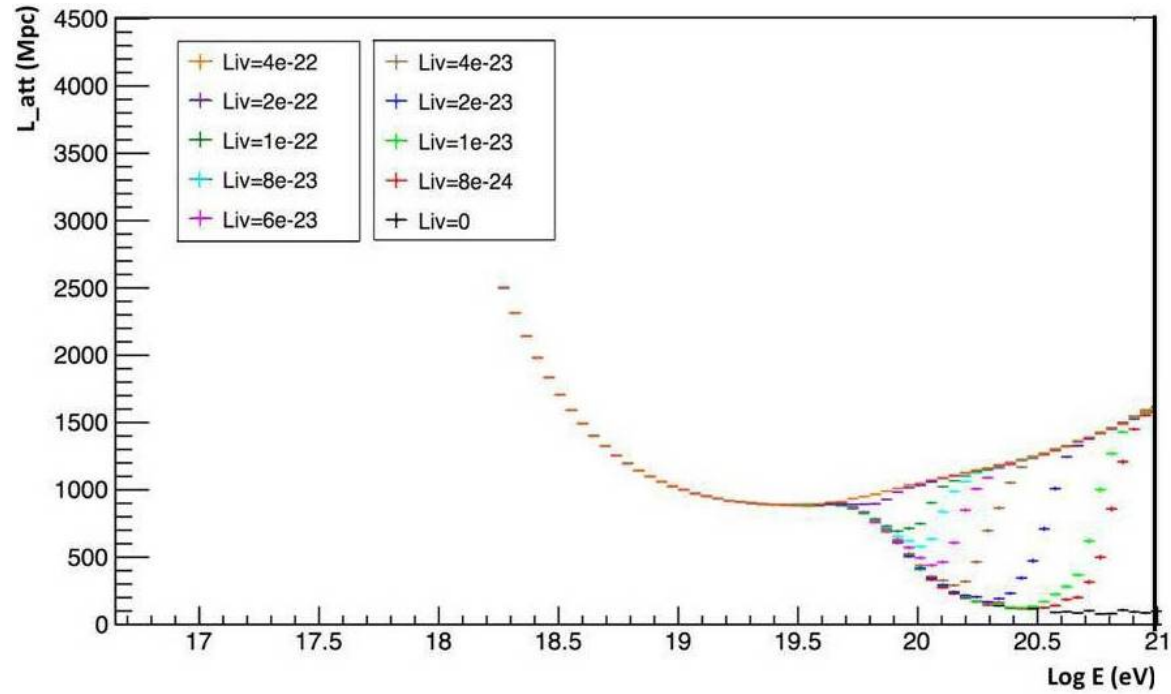

Figure 3. Attenuation length as a function of energy, plotted for ten different values of the LIV parameter.

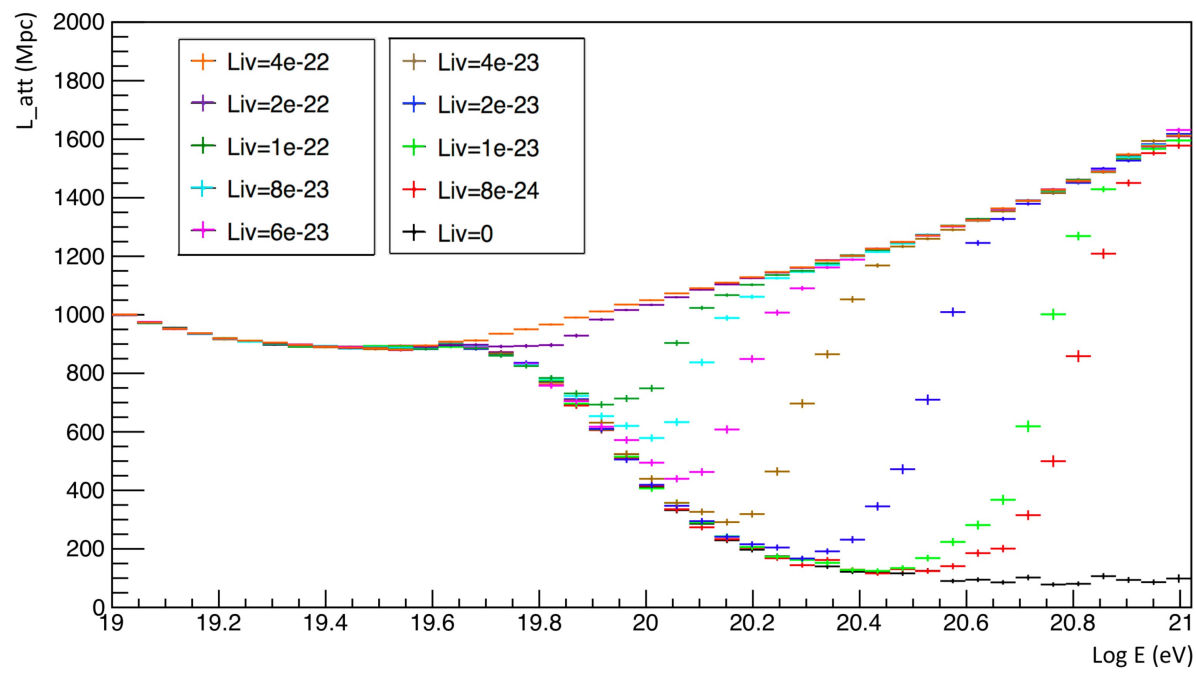

Figure 4. Attenuation length as a function of energy; blow up of the plot in Figure (4). 


\section{Conclusions}

In this work, we investigated the possibility of exploiting the UHECR physics to detect supposed signatures of the quantum structure of spacetime. Nowadays, the Lorentz covariance stands at the base of our physics knowledge, but QG can introduce small departures from the classic scenario. We studied these supposed departures simulating UHECRs propagation in a framework of Lorentz covariance modification. In this research, we generalize the results of previous works $[7,8]$ in the context of a more complete formulation of the HMSR model [26]. Indeed, in our proposal a way is outlined to generalize HMSR theory in curved spacetime introducing a formalism that defines the threshold energy of reactions in a modified covariant framework. The model foresees a minimal extension of the particle $S M$, preserves the usual gauge symmetry $S U(3) \otimes S U(2) \otimes U(1)$ and does not introduce exotic particles or reactions. The resulting geometry is a generalized Finsler spacetime and all the GZK cutoff modification effects have been computed in this context. We underline that the methodology here introduced can be used to set the stage of the model in the context of the more restrictive Finsler geometry and we have demonstrated that the inelasticity modification that underlies the opacity sphere enlargement can be still computed in the Finsler spacetime. Moreover, a generalized analysis framework for the possible QG induced effects is introduced investigating a more general form of the MDRs. Finally, we can state that all the presented results are obtained in the context of a more complete theoretical model formulation.

Our proposal is analogous to that of [4], but is based on a covariant formulation, such as in DSR theories [55,56]. The kinematical solution presented in our work is based on the hypothesis that the QG induced corrections are particle species depending and are caused by the particle interplay with the QG structure of spacetime. The introduced QG phenomenology is limited to the free particle kinematics since nowadays it is not totally clear how DSR theories can affect the process dynamics, since the related computations are incredibly complicated [57]. However, the kinematic perturbation here introduced can modify the allowed phase space for different particle interaction processes amending the related threshold energies. Hence, in this scenario QG may affect the interaction of different particle species, such as in the case of the GZK cut-off phenomenon.

The conducted simulations foresee an enlargement of the attenuation length in accordance with other works [5-8,58,59], but are obtained in a covariance-preserving scenario as proposed in [60].

We emphasize the importance of preserving covariance in the HMSR model, even if in an amended formulation, which allows the definition of relativistic kinematical invariants. As a direct consequence, it is possible to choose the most suitable reference frame to simplify the computations, suppressing some perturbation terms thanks to the involved energy scale ratios. Furthermore, in the astroparticle sector, covariance can be a great experimental advantage, since all the obtained data can be collected without the necessity to introduce any sidereal discrimination related to the orientation of the detector with respect to a fixed privileged reference frame.

The analysis strategy presented in this work can be improved including a heavier UHECR component, which is a more realistic CR composition, as indicated by the Pierre Auger collaboration [16]. The QG perturbations are expected to be larger for a CR component heavier than protons. Indeed, the kinematical perturbations can affect the propagation of bare nuclei in a more significant way, amending the threshold processes underlying the heavy UHECR photodissociation. This improvement of the analysis will be necessary since presently the measured UHECR composition suggests that the spectrum end is not caused only by the GZK cut-off.

Funding: This work was supported by the Fondazione Fratelli Giuseppe Vitaliano, Tullio e Mario Confalonieri-Milano, financing a post doctoral fellowship at Dipartimento di Fisica--Università degli Studi di Milano.

Informed Consent Statement: Not applicable. 
Data Availability Statement: Not applicable.

Acknowledgments: The author wants to thank Christian Pfeifer and Nicoleta Voicu for the useful discussion and advice to improve this work.

Conflicts of Interest: The authors declare no conflict of interest.

\section{Abbreviations}

The following abbreviations are used in this manuscript:

CR Cosmic Ray

UHECR Ultra-High-Energy Cosmic Ray

UHE Ultra-High-Energy

$\mathrm{CMB} \quad$ Cosmic Microwave Background

ELB Extragalactic Light Background

GMF Galactic Magnetic Field

EGMF Extragalactic Magnetic Field

SM Standard Model

SR Special Relativity

GR General Relativity

QG Quantum Gravity

SME Standard Model Extension

HMSR Homogeneously Modified Special Relativity

DSR Doubly Special Relativity

QFT Quantum Field Theory

\section{Appendix A}

In this appendix, we show that it is possible to choose perturbations $h$ and $k$, functions of the ratio $|\vec{p}| / E$, whose derivatives exhibit again a perturbative character. If we admit for these functions the general form:

$$
h\left(\frac{|\vec{p}|}{E}\right)=\sum_{n} \alpha_{n}\left(\frac{|\vec{p}|}{E}\right)^{n} \quad k\left(\frac{|\vec{p}|}{E}\right)=\sum_{n} \beta_{n}\left(\frac{|\vec{p}|}{E}\right)^{n},
$$

where the function magnitude is encoded in the coefficients $\alpha_{n} \ll 1$ and $\beta_{n} \ll 1$, hence it is possible to choose these coefficients in order to pose: $h, k \simeq O(1)$.

The definition domain of $h$ and $k$ is the interval $[0,1)$. Hence, requiring a strongly limited magnitude, even in the high-energy limit $|\vec{p}|, E \rightarrow+\infty$, one obtains the convergence of the series:

$$
\sum_{n} \alpha_{n}<+\infty, \quad \sum_{n} \beta_{n}<+\infty
$$

Therefore, the sequences $\alpha_{n}, \beta_{n}$ must decrease fast enough admitting the limit

$$
\alpha_{n}, \beta_{n} \rightarrow 0
$$

The derivatives of the functions $h$ and $k$ with respect to the variables $p_{i}$ and $E$ take the following form:

$$
\begin{aligned}
& \frac{\partial h(p)}{\partial p_{i}}=\sum_{n} n \alpha_{n} \frac{p_{i}|\vec{p}|^{n-2}}{E^{n}} \\
& \frac{\partial h(p)}{\partial E}=-\sum_{n} n \alpha_{n} \frac{|\vec{p}|^{n}}{E^{n+1}},
\end{aligned}
$$

where $i \in\{1,2,3\}$.

A simple check of the assertion can be obtained considering the $h$ and $k$ as linear, that is, series ending at the first expansion term. In this case, the magnitude order of the 
functions and their respective derivatives is determined by the coefficients $\alpha_{1}$ and $\beta_{1}$ and therefore present the same perturbation character.

In the more general case, the assertion can be proved as a direct consequence of these last equations, indeed it is possible to choose the coefficients $\alpha_{n}$ and $\beta_{n}$ such that even the derivatives of the functions $h$ and $k$ are limited in the definition domain and their magnitudes are determined again by the first coefficients of the expansions, since they belong to quickly decreasing sequences. The chosen perturbation functions $h$ and $k$ admit, therefore, derivatives with the same magnitude, proving the assertion. Some explicit examples are the linear function $\alpha(|\vec{p}| / E)$, the truncated exponential series $\exp (\alpha|\vec{p}| / E)-1$ and the trigonometric function $\sin (\alpha|\vec{p}| / E)$, with an ad hoc choice for the coefficient $\alpha$, which must be strongly limited and determines the perturbative character of the selected function.

The perturbative order of the matrix $A$ follows from a direct computation. Indeed, the entries of the matrix $A$ involve first and second-order derivatives of the perturbation functions $h$ and $k$ multiplied, respectively, by the momentum to the first or the second power.

\section{References}

1. Hillas, A.M. The origin of ultra-high-energy cosmic rays. Annu. Rev. Astron. Astrophys. 1984, 22, 425-444. [CrossRef]

2. Greisen, K. End to the cosmic ray spectrum? Phys. Rev. Lett. 1966, 16, 748-750. [CrossRef]

3. Zatsepin, G.; Kuzmin, V. Upper limit of the spectrum of cosmic rays. JETP Lett. 1996, 4, 78-80.

4. Coleman, S.R.; Glashow, S.L. High-energy tests of Lorentz invariance. Phys. Rev. D 1999, 59, 116008. [CrossRef]

5. Stecker, F.W.; Scully, S.T. Searching for New Physics with Ultrahigh Energy Cosmic Rays. New J. Phys. 2009, 11, 085003. [CrossRef]

6. Scully, S.; Stecker, F. Lorentz Invariance Violation and the Observed Spectrum of Ultrahigh Energy Cosmic Ray. Astropart. Phys. 2009, 31, 220-225. [CrossRef]

7. Torri, M.D.C.; Bertini, S.; Giammarchi, M.; Miramonti, L. Lorentz Invariance Violation effects on UHECR propagation: A geometrized approach. JHEAp 2018, 18, 5-14. [CrossRef]

8. Torri, M.D.C.; Caccianiga, L.; di Matteo, A.; Maino, A.; Miramonti, L. Predictions of Ultra-High Energy Cosmic Ray Propagation in the Context of Homogeneously Modified Special Relativity. Symmetry 2020, 12, 1961. [CrossRef]

9. Torri, M.D.C. Lorentz Invariance Violation Effects on Ultra High Energy Cosmic Rays Propagation, a Geometrical Approach. Ph.D. Thesis, Milan University, Milano, Italy, 2019.

10. Jansson, R.; Farrar, G.R. A new model of the galactic magnetic field. Astrophys. J. 2012, 757, 13. [CrossRef]

11. Jansson, R.; Farrar, G.R. The galactic magnetic field. Astrophys. Galaxies 2012, 761, L11. [CrossRef]

12. Neronov, A.; Vovk, I. Evidence for Strong Extragalactic Magnetic Fields from Fermi Observations of TeV Blazars. Science 2010, 328, 73-75. [CrossRef] [PubMed]

13. Ade, P.A.R.; Aghanim, N.; Arnaud, M.; Arroja, F.; Ashdown, M.; Aumont, J.; Baccigalupi, C.; Ballardini, M.; Banday, A.J.; Barreiro, R.B.; et al. Planck 2015 results. XIX. Constraints on primordial magnetic fields. Astron. Astrophys. 2016, 594, A19.

14. Sigl, G.; Miniati, F.; Ensslin, T.A. Ultrahigh energy cosmic rays in a structured and magnetized universe. Phys. Rev. D 2003, 68, 043002. [CrossRef]

15. Dolag, K.; Grasso, D.; Springel, V.; Tkachev, I. Constrained simulations of the magnetic field in the local Universe and the propagation of ultrahigh energy cosmic rays. J. Cosmol. Astropart. Phys. 2005, 2005, 009. [CrossRef]

16. Pierre Auger Collaboration.Depth of maximum of air-shower profiles at the Pierre Auger Observatory. II. Composition implications. Phys. Rev. D 2014, 90, 122006. [CrossRef]

17. Bellido, J. Depth of maximum of air-shower profiles at the Pierre Auger Observatory: Measurements above $10^{17.2} \mathrm{eV}$ and Composition Implications. In Proceedings of the 35th International Cosmic Ray Conference, ICRC2017, Busan, Korea, 12-20 July 2017; p. 506.

18. Batista, R.A.; Biteau, J.; Bustamante, M.; Dolag, K.; Engel, R.; Fang, K.; Kampert, K.H.; Kostunin, D.; Mostafa, M.; Murase, K.; et al. Open Questions in Cosmic-Ray Research at Ultrahigh Energies. Front. Astron. Space Sci. 2019, 6, 23. [CrossRef]

19. Colladay, D.; Kostelecký, V.A. Lorentz violating extension of the standard model. Phys. Rev. D 1998, 58, 116002. [CrossRef]

20. Kostelecky, V.A.; Russell, N. Data Tables for Lorentz and CPT Violation. Rev. Mod. Phys. 2011, 83, 11. [CrossRef]

21. Cohen, A.G.; Glashow, S.L. Very special relativity. Phys. Rev. Lett. 2006, 97, 021601. [CrossRef]

22. Amelino-Camelia, G. Doubly special relativity. Nature 2002, 418, 34-35. [CrossRef] [PubMed]

23. Amelino-Camelia, G. Doubly special relativity: First results and key open problems. Int. J. Mod. Phys. D 2002, 11, 1643. [CrossRef]

24. Amelino-Camelia, G.; Freidel, L.; Kowalski-Glikman, J.; Smolin, L. The principle of relative locality. Phys. Rev. D 2011, 84, 084010. [CrossRef]

25. Amelino-Camelia, G.; Bianco, S.; Rosati, G. Planck-Scale-Deformed Relativistic Symmetries and Diffeomorphisms on Momentum Space. Phys. Rev. D 2020, 101, 026018. [CrossRef]

26. Torri, M.; Antonelli, V.; Miramonti, L. Homogeneously Modified Special relativity (HMSR). Eur. Phys. J. C 2019, 79, 808. [CrossRef]

27. Aloisio, R.; Boncioli, D.; Grillo, A.; Petrera, S.; Salamida, F. SimProp: A Simulation Code for Ultra High Energy Cosmic Ray Propagation. JCAP 2012, 10, 007. [CrossRef] 
28. Magueijo, J.; Smolin, L. Gravity's rainbow. Class. Quant. Grav. 2004, 21, 1725. [CrossRef]

29. Magueijo, J.; Smolin, L. Lorentz invariance with an invariant energy scale. Phys. Rev. Lett. 2002, 88, 190403. [CrossRef]

30. Antonelli, V.; Miramonti, L.; Torri, M.D.C. Neutrino oscillations and Lorentz Invariance Violation in a Finslerian Geometrical model. Eur. Phys. J. C 2018, 78, 667. [CrossRef]

31. Torri, M.D.C. Neutrino Oscillations and Lorentz Invariance Violation. Universe 2020, 6, 37. [CrossRef]

32. Pfeifer, C.; Wohlfarth, M.N.R. Finsler geometric extension of Einstein gravity. Phys. Rev. D 2012, 85, 064009. [CrossRef]

33. Hohmann, M.; Pfeifer, C.; Voicu, N. Finsler gravity action from variational completion. Phys. Rev. D 2019, 100, 064035. [CrossRef]

34. Pfeifer, C. Finsler spacetime geometry in Physics. Int. J. Geom. Meth. Mod. Phys. 2019, 16, 1941004. [CrossRef]

35. Hohmann, M.; Pfeifer, C.; Voicu, N. Cosmological Finsler Spacetimes. Universe 2020, 6, 65. [CrossRef]

36. Javaloyes, M.A.; Sánchez, M. On the definition and examples of cones and Finsler spacetimes. RACSAM 2020, 114, 30. [CrossRef]

37. Bernal, A.; Javaloyes, M.Á.; Sánchez, M. Foundations of Finsler spacetimes from the Observers' Viewpoint. Universe 2020,6 , 55. [CrossRef]

38. Miron, R. Lagrangian and Hamiltonian Geometries. Applications to Analytical Mechanics. arXiv 2012, arXiv:1203.4101.

39. Vacaru, S.; Stavrinos, P.; Gaburov, E.; Gonta, D. Clifford and Riemann-Finsler structures in geometric mechanics and gravity. arXiv 2005, gr-qc/0508023.

40. Greenberg, O. Why is CPT fundamental? Found. Phys. 2006, 36, 1535-1553. [CrossRef]

41. Greenberg, O. CPT violation implies violation of Lorentz invariance. Phys. Rev. Lett. 2002, 89, 231602. [CrossRef] [PubMed]

42. Antonelli, V.; Miramonti, L.; Torri, M.D.C. Phenomenological Effects of CPT and Lorentz Invariance Violation in Particle and Astroparticle Physics. Symmetry 2020, 12, 1821. [CrossRef]

43. Girelli, F.; Liberati, S.; Sindoni, L. Planck-scale modified dispersion relations and Finsler geometry. Phys. Rev. D 2007, 75, 064015. [CrossRef]

44. Kostelecky, A. Riemann-Finsler geometry and Lorentz-violating kinematics. Phys. Lett. B 2011, 701, 137. [CrossRef]

45. Edwards, B.R.; Kostelecky, V.A. Riemann-Finsler geometry and Lorentz-violating scalar fields. Phys. Lett. B 2018, 786, 319. [CrossRef]

46. Lämmerzahl, C.; Perlick, V. Finsler geometry as a model for relativistic gravity. Int. J. Geom. Meth. Mod. Phys. 2018, 15 (Suppl. 1), 1850166. [CrossRef]

47. Axiomatic formulations of modied gravity theories with nonlinear dispersion relations and Finsler Lagrange Hamilton geometry. Eur. Phys. J. C 2018, 78, 969. [CrossRef]

48. Schreck, M. Classical Lagrangians and Finsler structures for the nonminimal fermion sector of the Standard-Model Extension. Phys. Rev. D 2016, 93, 105017. [CrossRef]

49. Seiberg, N.; Witten, E. String theory and noncommutative geometry. JHEP 1999, 9, 32. [CrossRef]

50. Carroll, S.M.; Harvey, J.A.; Kostelecky, V.A.; Lane, C.D.; Okamoto, T. Noncommutative field theory and Lorentz violation. Phys. Rev. Lett. 2001, 87, 141601. [CrossRef]

51. Stecker, F.W. Photodisintegration of ultrahigh-energy cosmic rays by the universal radiation field. Phys. Rev. 1969, 180, 1264-1266. [CrossRef]

52. Abbasi, R.U.; Abu-Zayyad, T.; Allen, M.; Amman, J.F.; Archbold, G.; Belov, K.; Belz, J.W.; Ben Zvi, S.Y.; Bergman, D.R.; Blake, S.A.; et al. First observation of the Greisen-Zatsepin-Kuzmin suppression. Phys. Rev. Lett. 2008, 100, 101101. [CrossRef]

53. Abraham, J.; Abreu, P.; Aglietta, M.; Aguirre, C.; Allard, D.; Allekotte, I.; Allen, J.; Allison, P.; Alvarez-Muniz, J.; Ambrosio, M.; et al. Observation of the suppression of the flux of cosmic rays above $4 \times 10^{19} \mathrm{eV}$. Phys. Rev. Lett. 2008, 101, 061101. [CrossRef] [PubMed]

54. Batista, R.A.; Boncioli, D.; di Matteo, A.; van Vliet, A.; Walz, D. Effects of uncertainties in simulations of extragalactic UHECR propagation, using CRPropa and SimProp. JCAP 2015, 10, 063. [CrossRef]

55. Amelino-Camelia, G.; Palmisano, M.; Ronco, M.; D'Amico, G. Mixing coproducts for theories with particle-dependent relativistic properties. Int. J. Mod. Phys. D 2020, 29, 2050017. [CrossRef]

56. Amelino-Camelia, G. Particle-Dependent Deformations of Lorentz Symmetry. Symmetry 2012, 4, 344-378. [CrossRef]

57. Amelino-Camelia, G.; Arzano, M. Coproduct and star product in field theories on Lie algebra noncommutative space-times. Phys. Rev. D 2002, 65, 084044. [CrossRef]

58. Bietenholz, W. Cosmic Rays and the Search for a Lorentz Invariance Violation. Phys. Rept. 2011, 505, 145-185. [CrossRef]

59. Mattingly, D. Modern tests of Lorentz invariance. Living Rev. Relativ. 2005, 8, 1-84. [CrossRef]

60. Amelino-Camelia, G. Kinematical solution of the UHE-cosmic—ray puzzle without a preferred class of inertial observers. Int. J. Mod. Phys. D 2003, 12, 1211-1226. [CrossRef] 